\title{
Bi-Directional Recurrent Attentional Topic Model
}

\author{
SHUANGYIN LI, South China Normal University \\ YU ZHANG, Southern University of Science and Technology \\ RONG PAN, Sun Yat-sen University
}

In a document, the topic distribution of a sentence depends on both the topics of its neighbored sentences and its own content, and it is usually affected by the topics of the neighbored sentences with different weights. The neighbored sentences of a sentence include the preceding sentences and the subsequent sentences. Meanwhile, it is natural that a document can be treated as a sequence of sentences. Most existing works for Bayesian document modeling do not take these points into consideration. To fill this gap, we propose a bi-Directional Recurrent Attentional Topic Model (bi-RATM) for document embedding. The bi-RATM not only takes advantage of the sequential orders among sentences but also uses the attention mechanism to model the relations among successive sentences. To support to the bi-RATM, we propose a bi-Directional Recurrent Attentional Bayesian Process (bi-RABP) to handle the sequences. Based on the bi-RABP, bi-RATM fully utilizes the bidirectional sequential information of the sentences in a document. Online bi-RATM is proposed to handle large-scale corpus. Experiments on two corpora show that the proposed model outperforms state-of-the-art methods on document modeling and classification.

CCS Concepts: • Information systems $\rightarrow$ Document topic models; • Computing methodologies $\rightarrow$ Topic modeling; Natural language processing; Learning in probabilistic graphical models;

Additional Key Words and Phrases: Bi-directional recurrent attentions, recurrent attentional Bayesian process, topic modeling

ACM Reference format:

Shuangyin Li, Yu Zhang, and Rong Pan. 2020. Bi-Directional Recurrent Attentional Topic Model. ACM Trans. Knowl. Discov. Data 14, 6, Article 74 (September 2020), 30 pages.

https://doi.org/10.1145/3412371

This work was supported by the Key-Area Research and Development Program of Guangdong Province (No. 2019B010137003), National Key-Area Research and Development Program of China (No. 2018YFB1404402), Basic and Applied Basic Research Fund of Guangdong Province (No. 2019B1515120085), and Guangdong Science and Technology Fund (No. 2018A07071702).

Authors' addresses: S. Li (corresponding author), School of Computer Science, South China Normal University, Key Lab on Cloud Security and Assessment Technology of Guangzhou, No. 55, West of Zhongshan Avenue, Tianhe District, Guangzhou, Guangdong, 510631, China; email: shuangyinli@scnu.edu.cn; Y. Zhang, Department of Computer Science and Engineering, Southern University of Science and Technology, 1088 Xueyuan Avenue, Shenzhen, Guangdong, 518055, China; email: yu.zhang.ust@gmail.com; R. Pan, Department of Data and Computer Science, Sun Yat-sen University, Waihuan East Road 132, Guangzhou, Guangdong, 510006, China; email: panr@sysu.edu.cn.

Permission to make digital or hard copies of all or part of this work for personal or classroom use is granted without fee provided that copies are not made or distributed for profit or commercial advantage and that copies bear this notice and the full citation on the first page. Copyrights for components of this work owned by others than ACM must be honored. Abstracting with credit is permitted. To copy otherwise, or republish, to post on servers or to redistribute to lists, requires prior specific permission and/or a fee. Request permissions from permissions@acm.org. (c) 2020 Association for Computing Machinery.

1556-4681/2020/09-ART74 \$15.00

https://doi.org/10.1145/3412371 


\section{INTRODUCTION}

In the Internet era, there has been a huge amount of documents in many web applications, such as search engines, e-commerce websites, and mobile applications. Modeling documents into a vector space is one important task in machine learning. How to take advantage of elements in documents, such as words, sentences, and paragraphs, needs careful consideration. In general, documents are composed of a group of words with different frequencies, which can be modeled via the "bagof-words" assumption. Many works focus on extracting better representations for documents in many real-world applications. Probabilistic topic models provide a suite of algorithms to obtain good representations when facing a collection of documents. The representation obtained by a topic model often corresponds to latent topics in an interpretable space, which is an advantage over other models. Topic models have improved document classification and information retrieval [72] on unstructured text, and many models have been extended to the structured text/non-text data in computer vision [23] and collaborative filtering [42]. Topic models usually assume that words are interchangeable, which is helpful for efficient inference on large corpora [4]. Actually, documents are sequences of words, sentences, and paragraphs in a hierarchical manner and some works have modeled a document as a sequence of words, including the $n$-gram language modeling [10] and recurrent neural networks for language modeling [24, 64].

Although topic models have been widely used for document modeling, the topic coherence and the semantic coherence between sentences, which do exist in natural language, are ignored in existing works. In a coherent document, each sentence relates clearly to its neighbored sentences over latent topic space. That is, if a document is coherent, each sentence flows smoothly into the next sentence, which makes the structure of ideas or arguments clear to read. To see this, let us consider the following seven sentences describing "Machine Learning" from the Wikipedia:

(1) Machine learning $(M L)$ is a field of artificial intelligence that uses statistical techniques to give computer systems the ability to "learn" from data, without being explicitly programmed.

(2) Machine learning is employed in a range of computing tasks where designing and programming explicit algorithms with good performance is difficult or infeasible.

(3) Machine learning is closely related to computational statistics, which also focuses on predictionmaking through the use of computers.

(4) It has strong ties to mathematical optimization, which delivers methods, theory and application domains to the field.

(5) Machine learning is sometimes conflated with data mining, where the latter subfield focuses more on exploratory data analysis and is known as unsupervised learning.

(6) Within the field of data analytics, machine learning is a method used to devise complex models and algorithms that lend themselves to prediction.

(7) These analytical models allow researchers, data scientists, engineers, and analysts to "produce reliable, repeatable decisions and results" and uncover "hidden insights" through learning from historical relationships and trends in the data.

Obviously, sentence (5) is about the relationship between machine learning and data mining, whose topics are highly coherent with those of the three preceding sentences and so the topics in a sentence could recurrently affect the following sentences. Meanwhile, the topics of sentence (5) are also highly coherent with the following sentences. Without considering the detailed transitional expressions in linguistics, we can see that the semantics among the sentences are consistent.

From this example, we can get two aspects of inspiration. First, it is obvious that the sequences of sentences exist in the documents, and only considering the word information is obviously not enough if the sequential information among sentences is available. Meanwhile, a document can be treated as two-directional sequences of sentences, namely, the forward and backward direction. The bi-sequential property of sentences reflects on the topic coherence among sentences, where 
the topics can be extracted by topic models. Second, in the above example, the topics of the sentence (5) are more relevant to those of sentences (1) and (6) since they all mention data mining and machine learning. Thus, besides the topic coherence among sentences, it is intuitive that a sentence is related to the neighbored sentences with different weights. Thus, how to model the sequential property among sentences together with the weight signals in a document for the topic coherence on the sentence level is an interesting and meaningful task in the infrastructure of topic models.

Deep neural network turn out to be a powerful method to embed documents into a computable vector space, such as Replicated Softmax Model (RSM) [29] and over-RSM [52] based on convolutional neural networks $[34,59,65]$. With taking full advantage of the sequence information where documents are treated as sequences of words, recurrent neural networks (RNNs) and its variants such as long short-term memory (LSTM) have achieved great success on document modeling. Also, the attentional mechanism has been used in deep neural networks to capture the information of the main parts in images or documents. The attentional mechanism in deep neural networks is inspired by the visual attention mechanism found in humans, where persons are essentially able to focus more on a certain region of an image or document than other regions to extract the useful information.

Thus, it is intuitive and meaningful to adapt the attentional mechanism to model the bidirectional sequences of sentences under the framework of topic models. However, there are very few works considering the attentional mechanism in topic models and even in Bayesian modeling. To fill this gap, we propose a bi-Directional Recurrent Attentional Bayesian Process (bi-RABP), which can handle sequential data and allow a local recurrent information transmission through a sequence. The bi-RABP is extended from RABP, which models the sequential data in a recurrent manner with attentional signals, proposed by [38]. Different from RABP, bi-RABP considers the two-directional information of sequential data in a single Bayesian process. Then a document can be treated as a bi-directional sequential data in the sentence level and as a bag-of-words in the word level. Based on the proposed bi-RABP, we propose a bi-directional Recurrent Attentional Topic Model (bi-RATM). The bi-RATM can model sequences of sentences by considering the dependency between sentences as well as attention signals from two sequential directions. To the best of our knowledge, there is no work to consider sentence coherence and attention signals in Bayesian modeling. Also, we devise an online learning algorithm for the proposed bi-RATM to handle large-scale documents. Specifically, the main contributions of this work are four-fold:

(1) We propose a novel bi-RABP to model sequential data from two directions. The Bayesian process handles the sequential data with a local recurrent information transmission and an attention mechanism, which can produce great advantages of modeling the sequential data under the framework of topic models, especially for the bi-directional sequences of text data.

(2) A novel bi-RATM, which is a unified probabilistic language model of bi-directional sequences of sentences, is proposed to tackle the issue of document modeling. Based on the bi-RABP, the proposed bi-RATM fully takes advantage of the bi-directional sentences to learn high-quality document representations. Experiments have demonstrated that the proposed bi-RATM can achieve the state-of-the-art performance on document modeling and document classification tasks.

(3) With the proposed bi-RATM, we establish a previously unexplored connection between recurrent Bayesian methods and the attention signals, where the attention signals are adaptively learned for two-directional sequences of sentences. The attention mechanism used in the Bayesian methods can improve the capacity of modeling documents with bidirectional sequences of sentences. 
(4) Efficient variational inference is developed together with an online algorithm for bi-RATM to handle large-scale documents. This online algorithm allows bi-RATM to handle stream documents, which can make it efficient and easy to use on the large-scale corpus.

The rest of the article is organized as follows. In Section 2, we discuss the related works. In Section 3, we introduce the bi-RABP. In Section 4, we present the bi-RABP and the bi-RATM in detail. The inference method is developed in Section 5. In Section 7, we analyze the proposed bi-RATM and discuss the relations between the bi-RATM and the existing models. The online learning algorithm for the proposed bi-RATM is presented in Section 6, and the complexity analysis is given in Section 8. In Section 9, we present the experimental results on two corpora for document modeling and classification along with the experiments on online learning. Also, we show some case studies of attention signals among sentences. Finally, we conclude the whole article and discuss some future works in Section 10.

\section{RELATED WORKS}

To date, many models are proposed to handle various types of data, such as videos, images [70], and documents in many tasks, for example, object detection [2], content detection [71], data management [73, 78], and representation learning for words, sentences and documents [33, 38, 46, 53]. The methodologies of representation learning for documents mainly include undirected graphical models or directed graphical models. For the undirected graphical models, RSM uses a two-layer undirected graphical model to produce distributed representations of documents, which can be used to model and extract low-dimensional latent semantic representations from a collection of unstructured documents. [52] a deep Boltzmann machine called the over-RSM, which extracts distributed semantic representations from unstructured documents. Recently, many approaches based on neural networks are proposed to handle a collection of unlabeled documents [35], or data consisting of multiple modalities [61, 62].

As directed graphical models, many probabilistic topic models have been proposed [6, 7, 9, 30, $31,43]$. These models and their extensions have been applied to many tasks such as information retrieval [72], document classification [11], as well as the learning framework [55]. Some models, such as Latent Dirichlet Allocation (LDA) [7] and Correlated Topic Model (CTM) [6], are used to model unstructured documents with assumptions that the words in a document arise from a mixture of latent topics and that each topic is a distribution over the vocabulary. Many topic models such as $[26,27]$ take the order of words and the syntactic of sentences into consideration. [26] focus on short-range syntactic dependencies and long-range semantic dependencies between words. The Hidden Topic Markov Model (HTMM) proposed in [27] models the topics of words in a document as a Markov chain, which assumes that all words in the same sentence have the same topic and that successive sentences are more likely to have the same topics. The syntactic topic model proposed in [9] generates words via both thematically and syntactically constraints among them. Almost all the existing topic models consider the sequentiality of documents on the word level only. The Sequential LDA (SeqLDA) [21] considers the underlying sequential structure, i.e., a document consisting of multiple segments such as chapters and paragraphs via a hierarchical two-parameter Poisson-Dirichlet process [54]. However, different from the proposed method, SeqLDA does not consider attentions among segments. Recently, many researchers are interested in Lifelong topic model, which is a paradigm for never-ending topic learning $[15,16,74]$.

RNNs [64] provide an efficient way to handle the sequentiality of documents on both the word [44, 45] and the sentence levels, and they have been applied to various tasks, including machine translation [3], summarization [56], dialog systems [58], document modeling [40], and so on. The recurrent mechanism in neural networks has achieved great success in language 
modeling. However, there are few works on topic modeling to study the recurrent mechanism. Several Bayesian models use the recurrent mechanism to handle the topic dynamic in a corpus or document steams $[1,22]$.

There are some works $[25,28,77]$ which combine Bayesian models with deep neural networks. [25] present a deep graphical model, which selects topics via a Poisson factor analysis (PFA) model in its bottom binary layer, based on a deep sigmoid belief network or restricted Boltzmann machine. The deep Poisson factor model [28] is based on PFA, which applies a Poisson distribution to model observed vectors of counts. Gamma belief network [77] can be applied to handle the text corpora, where each document is represented as a term-frequency count vector. These models are kinds of deep Bayesian models to handle the text directly without considering more detailed information in text, such as the sequential information of the sentences in a document. There are also some works to build topic models with neural networks [18, 37, 51]; however, word embeddings are needed in these models. [19] propose a TopicRNN model which incorporates the RNN into latent topic models. It captures local (syntactic) dependencies using an RNN and global (semantic) dependencies using latent topics. Differently, TopicRNN models the sequential information of the words in a document, while in this work, we consider the recurrent property at the sentence level.

Attention signals are widely applied to language modeling for many text mining tasks $[3,41,56$, 58] and speech tasks [17]. However, all these models with the attention mechanism are under the neural network framework and few Bayesian models focus on language modeling with attention signals. Thus, in our article, we aim to incorporate the attention mechanism into the bi-directional recurrent Bayesian topic model for documents. For training models on large-scale data, various methods such as $[13,14,30,39,48,60,76]$ are investigated. We also propose an online learning algorithm to handle large-scale document streams.

\section{BI-DIRECTIONAL RECURRENT ATTENTIONAL BAYESIAN PROCESS}

\subsection{Recurrent Chinese Restaurant Process}

The recurrent Chinese restaurant process (RCRP) [1] is an extension of the Chinese restaurant process, known as the Dirichlet process (DP), which defines a distribution over a Dirichlet distribution [5]. The RCRP can be viewed as a discrete-time sequence of DPs, where the DP appearing later in time can have a certain probability to follow a previous DP, and meanwhile, it also has a probability to create a new Dirichlet distribution. Taking the generation of a dynamic corpus over time as an example, the topics in the corpus evolve over time and at time $t$, the topic distribution of the corpus $\theta_{t}$ reuses the topic distribution from time 1 to time $t-1$ with a certain probability. Also, at time $t, \theta_{t}$ can be drawn from $G_{0}(\theta)$ with a probability, where $G_{0}(\theta)$ is a base distribution over a given space $\theta \in \Theta$. Meanwhile, each element $\theta_{t, k}$ in $\theta_{t}$ is drawn from a standard DP. After that, we generate topics for each document over $\theta_{t}$ and generate words given the topics. The main extension of RCRP from DP is to consider the evolution of distributions over time, which takes into account the temporal coherence of documents [1]. The RCRP can be used as a prior for parameters of clustering models, such as a combination of RCRP and a bag-of-words model for document modeling shown in Figure 1(a).

However, in RCRP, it is not clear how to choose the time window for each time $t$. Furthermore, $\theta_{t}$ is highly correlated with a limited number of preceding time points. For example, in the sequential generation of sentences in a document, the topic distribution of current sentence is obviously highly correlated with several preceding sentences. Moreover, in [22], the temporal dependence of each time point is handcrafted and it keeps fixed for different time points. Such design may not handle different importances among the preceding time points for the current time point. Thus, in the next section we propose a novel recurrent attentional Bayesian process (RABP) to address these drawbacks of RCRP when modeling documents at the sentence level. 


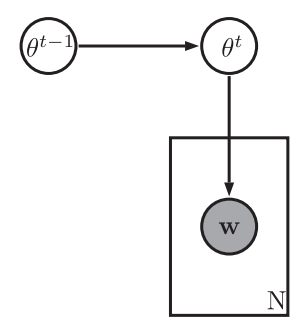

(a)

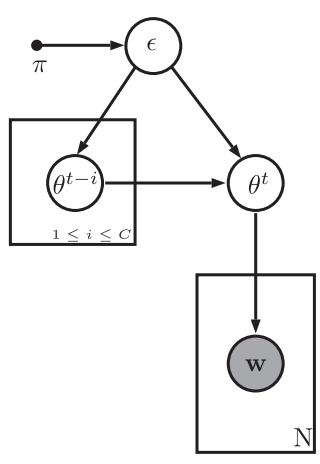

(b)

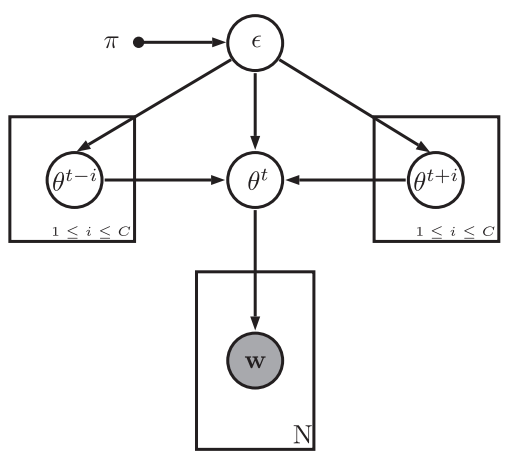

(c)

Fig. 1. (a): The RCRP combined with the bag-of-words assumption. (b): The RABP with the bag-of-words assumption. (c): The bi-RABP, an extension of the RABP. The shaded circles denote observed words and others are the hidden variables. $\epsilon$ denotes the attention signal, $\pi$ is a Dirichlet parameter, and $C$ is the length of time window.

\subsection{Recurrent Attentional Bayesian Process}

A RABP, denoted by $\operatorname{RABP}\left(G_{0}, \pi\right)$, is parameterized by a base measure $G_{0}$ and a concentration parameter $\pi$. The generative process for the RABP is as follows:

(1) Draw $\theta^{1}$ from $G_{0}$.

(2) For $t>1$

(a) Draw $\epsilon=\left(\epsilon_{1}, \ldots, \epsilon_{C+1}\right)^{T}$ from $\operatorname{Dir}(\pi)$, where $\operatorname{Dir}(\pi)$ denotes a Dirichlet distribution with parameter $\pi$

(b) With probability $\epsilon_{i}$, draw $\theta^{t}$ from $\delta_{\theta^{t-i}}$ for $i=1, \ldots, C$, where $\delta_{a}$ denotes a discrete distribution whose probability mass function is equal to 1 at the point $a$;

(c) With probability $\epsilon_{C+1}$, draw $\theta^{t}$ from $G_{0}$.

In this generative process, $G_{0}$ is the base distribution and $C$ is the length of the time window. Here $\epsilon=\left(\epsilon_{1}, \ldots, \epsilon_{C+1}\right)$, a $(C+1)$-dimensional vector, is defined as the attention signal. It captures the importance of a preceding neighbor $\theta^{t-i}$ to $\theta^{t} . \epsilon$ satisfies $\sum_{i=1}^{C+1} \epsilon_{i}=1$ as it follows a Dirichlet distribution with parameter $\pi$. The graphical model of RABP is shown in Figure 1(b).

Based on the generative process, $\theta^{t}$ can be represented as

$$
\theta^{t} \mid \theta^{t-C: t-1}, G_{0}, \pi \sim \sum_{i=1}^{C} \epsilon_{i} \cdot \theta^{t-i}+\epsilon_{C+1} \cdot G_{0}
$$

where $K$ is the length of each $\theta^{i}$ and $\theta^{j-C: j-1}$ is a $C \times K$ matrix containing $C$ preceding parameters. The attention signal $\epsilon_{i}$ reflects the importance of a preceding parameter in the sequence to the current one.

The RABP is partially related to the RCRP and Dirichlet-Hawkes Process (DHP) [22] which models the rate intensity and diversity of the temporal evolution in a continuous timeline. The main difference between the RABP and RCRP is that the RABP considers several preceding time points with dynamic attentional weights, while in the RCRP the dependency of the parameter over the preceding ones is invariant to both the positions and the content information. The DHP focuses on modeling the intensity of discrete events via a Hawkes process, but the RABP can model recurrent sequences in a discrete space with attention signals. The RABP considers the 
local reflection for the current state and can be used as a prior to model documents as sequences of sentences, which will be elaborated in the next section.

\subsection{Bi-Directional Recurrent Attentional Bayesian Process}

We present the bi-RABP, which is an extension of the RABP. The bi-RABP aims to model the sequential data from two directions. Based on the above definition of RABP, $\theta^{t}$ in bi-RABP $\left(G_{0}, \pi\right)$ can be represented as:

$$
\theta^{t} \mid \theta^{t-C: t-1}, \theta^{t+1: t+C}, G_{0}, \pi \sim \sum_{i=1}^{C} \epsilon_{i} \cdot \theta^{t-i}+\sum_{j=1}^{C} \epsilon_{j+C} \cdot \theta^{t+j}+\epsilon_{2 C+1} \cdot G_{0}
$$

where the second term measures the influence of the backward directional sequence for $\theta^{t}$. The graphical model of bi-RABP is shown in Figure 1(c). The forward and backward sequences have the same length of time window for the ease of presentation and it is easy to extend to different lengths in the two directions. Note that, the two different directional sequences share a joint attentional signal vector, $\epsilon$ and the dimension of $\epsilon$ is $2 \mathrm{C}+1$.

Comparing with the RABP, the main improvement of the bi-RABP is that it generates the current node jointly by the preceding and subsequent nodes at the same time point. This operation could improve the ability to capture the local information from two directions for the sequential data, such as textual data.

\section{BI-DIRECTIONAL RECURRENT ATTENTIONAL TOPIC MODEL}

The topic distribution of a sentence is highly related to those of preceding sentences and subsequent sentences, which is called cohesion or coherence in linguistics. From the forward direction, the topics of the sentence are affected by the preceding sentences, which matches the motivation of the RABP. From the backward direction, the topics of the sentence are also affected by the subsequent sentences, which can be modeled by another RABP. Hence, it is reasonable that the topics of the sentence are affected by both preceding sentences and subsequent sentences, where the jointly attentional signal should be considered.

Thus, we can model a document as a bi-directional sequence of sentences with the proposed bi-RABP, leading to the proposed bi-RATM, which attempts to capture the joint influence of proceeding sentences and subsequent sentences to the current one with unitized attentional signals. Moreover, the topics of a sentence are also affected by those of the host document that the sentence belongs to. Hence, the topic distribution of a sentence is generated from those of both its preceding and subsequent sentences as well as the host document. For each sentence, many models can be used and here we use a simple bag-of-words language model, i.e., LDA.

As a collection of $M$ documents, a corpus is defined as $D=\left\{\mathbf{d}^{1}, \ldots, \mathbf{d}^{M}\right\}$, where $\mathbf{d}^{i}(i \in$ $\{1, \ldots, M\})$ denotes the $i$-th document. A document $\mathrm{d}^{i}$, denoted by $\mathrm{d}^{i}=\left(\mathrm{s}_{1}^{i}, \ldots, \mathrm{s}_{S_{i}}^{i}\right)$, is a sequence of $S_{i}$ sentences, where $s_{j}^{i}\left(j \in\left\{1, \ldots, S_{i}\right\}\right)$ denotes the $j$-th sentence in $\mathbf{d}^{i}$. Let $\mathbf{s}_{j}^{i}=\left(w_{j, 1}^{i}, \ldots, w_{j, N_{j}^{i}}^{i}\right)$ denote the vector of $N_{j}^{i}$ words associated with sentence $\mathbf{s}_{j}^{i}$.

Let $\theta^{s_{j}}$ denote the topic distribution of the $j$-th sentence $\mathbf{s}_{j}$ in document $\mathbf{d}$, which is the topic distribution over $K$ latent topics. Based on the bi-RABP, $\theta^{s_{j}}$ is generated by $\theta^{t-C: t-1}, \theta^{t+1: t+C}$ and $G_{0}$ following Equation (2). Thus, the generation process of bi-RATM is defined as follows:

(1) For each topic $k \in\{1, \ldots, K\}$, draw $\beta_{k} \sim \operatorname{Dir}(\mu)$, where $\mu$ is a $V$-dimensional parameter vector of $\beta$;

(2) For each document $\mathbf{d}^{i}, i \in\{1, \ldots, M\}$ : 


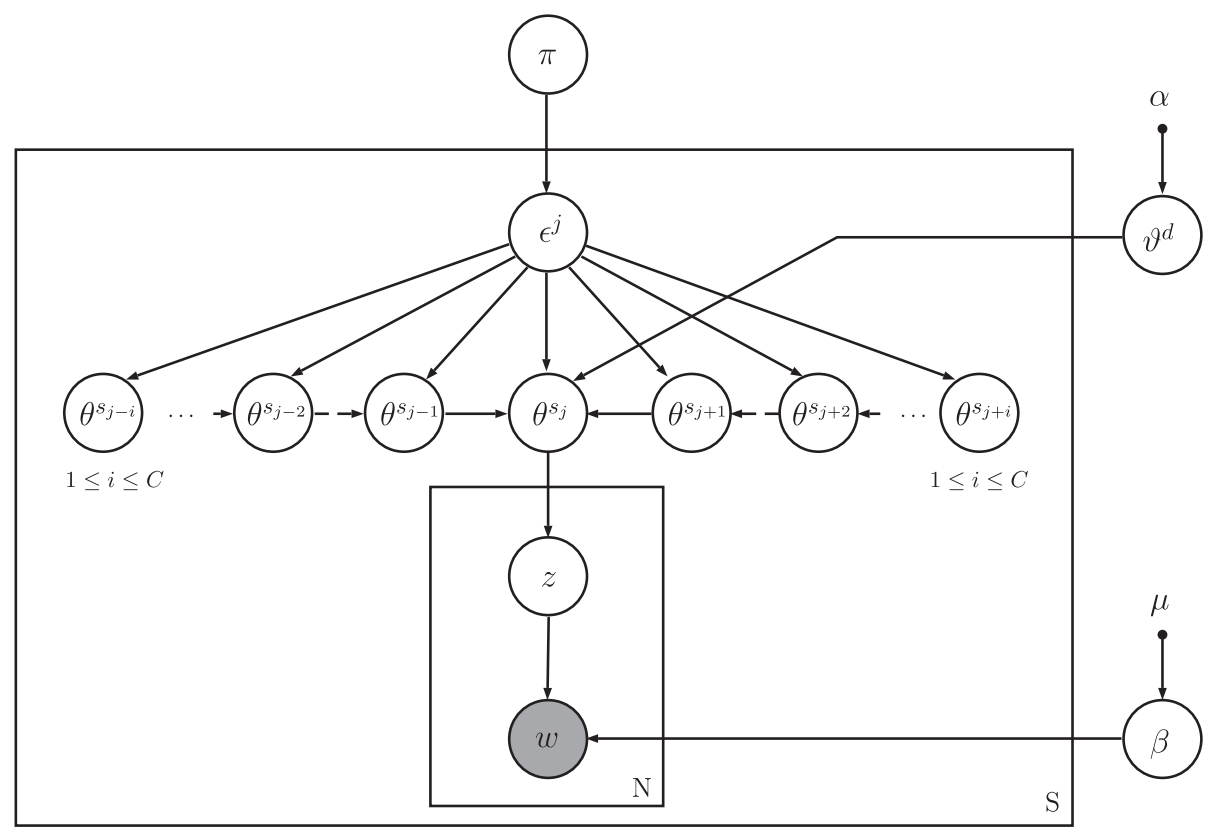

Fig. 2. The graphical model of the bi-RATM. $\vartheta^{d}$ is the topic distribution of the document $d . \theta^{s_{j-i}}$ denotes the topic distribution of a preceding sentence, and $\theta^{s_{j+i}}$ denotes the topic distribution of a subsequent sentence where $1 \leq i \leq C$. The dimension of the joint attentional vector is $2 C+1$.

(a) Draw $\vartheta^{d} \sim \operatorname{Dir}(\alpha)$;

(b) For the sentence $\mathbf{s}_{j}, j \in\left\{1, \ldots, S_{i}\right\}$ in the document $\mathbf{d}$ :

(i) Draw $\theta^{s_{j}} \sim \operatorname{bi}-\operatorname{RABP}\left(\delta_{\vartheta d}, \pi\right)$;

(ii) For each word $w_{n}, n \in\left\{1, \ldots, N_{j}\right\}$ in sentence $s_{j}$ :

(A) Draw $z_{n} \sim \operatorname{Mult}\left(\theta^{s_{j}}\right)$;

(B) Draw $w_{n} \sim \operatorname{Mult}\left(\beta_{z_{n}}\right)$.

In this generative process, $\vartheta^{d}$, a $K$-dimension vector following a Dirichlet distribution, describes the topic distribution of a document and it is used as $G_{0}$ in the bi-RABP for the topics of sentences. A topic denoted by $\beta_{k}$ is a distribution over a fixed vocabulary. $\mu$ is a Dirichlet prior of $\beta$.

Joint attention signals are used in the bi-RABP without explicitly introducing in the generative process and note that attention signals are adaptive in different sentences. $z_{n}$ is the topic assignment for each word $n$ and it describes the topic distribution of a word. $\theta^{s_{j}} \sim \operatorname{bi}-\operatorname{RABP}\left(\delta_{\vartheta}, \pi\right)$ indicates that the topic distribution of current sentence $\mathbf{s}_{j}$ is generated by a bi-RABP, which means that sentence $s_{j}$ depends on $C$ preceding sentences and the $C$ subsequent sentences via adaptive attention signals, $\epsilon^{j}$, as described in bi-RABP. Figure 2 shows the graphical model of the bi-RATM.

\section{MODEL INFERENCE}

The main problem in the inference for the bi-RATM is to estimate the posterior distribution of latent variables conditioned on observed data. Given a sentence $s_{j}$ with $N_{j}$ words in document $d$, we can easily get the posterior distribution of the latent variables in the proposed model as:

$$
p\left(\varepsilon^{j}, \mathbf{z} \mid \mathbf{s}_{j}, \beta, \pi, \alpha\right)=\frac{p\left(\varepsilon^{j}, \mathbf{z}, \mathbf{s}_{j} \mid \beta, \pi, \alpha\right)}{p\left(\mathbf{s}_{j} \mid \beta, \pi, \alpha\right)} .
$$


Typical topic models can be learned by Gibbs sampling methods due to the conjugate property between the distribution of the topic assignment and the prior over the topic distribution. However, the bi-RATM does not enjoy such property due to the prior for the topic distribution of a sentence (see Equation (2)), making the posterior distribution in the bi-RATM intractable to compute. Thus, we resort to the variational inference to approximate the posterior of a sentence to minimize the Kullback-Leibler (KL) divergence from the variational distribution to the true posterior distribution $[32,68]$. The variational method casts the inference problem as an optimization problem to approximate the posterior distribution [30]. [43] shows that minimizing the KL divergence between the variational posterior probability and the true posterior probability is equivalent to maximizing the Evidence Lower Bound (ELBO). In the variational inference, the posterior distribution is approximated by the product of a group of variational distributions with free variational parameters and the group of variational distributions are enforced to be close to the true posterior.

For each sentence $s_{j}$ with $N_{j}$ words in document $d$, we use the following fully-factorized variational distribution as

$$
q\left(\vartheta^{d}, \epsilon^{j}, \mathbf{z} \mid \rho^{d}, \xi, \gamma\right)=q\left(\vartheta^{d} \mid \rho^{d}\right) \prod_{j=1}^{S_{i}} q\left(\epsilon^{j} \mid \xi\right) \prod_{n=1}^{N_{j}} q\left(z_{n} \mid \gamma_{n}\right),
$$

where $\rho^{d}$ is a variational parameter of a Dirichlet distribution for the document $d, \xi$ is a variational parameter of a Dirichlet distribution for sentence $s_{j}$, and $\gamma_{n}$ is a variational parameter of a multinomial distribution.

Thus, the Jensen's lower bound on the log probability of the document $d$ can be computed as

$$
\mathcal{L}^{\mathrm{d}}\left(\beta, \pi, \vartheta^{d} ; \xi, \gamma, \rho^{d}\right)=\sum_{j=1}^{S_{i}} \mathcal{L}^{s_{j}}(\beta, \pi ; \xi, \gamma)+\mathbb{E}_{q}\left[\log p\left(\vartheta^{d} \mid \alpha\right)\right]-\mathbb{E}_{q}\left[\log q\left(\rho^{d}\right)\right],
$$

where the log probability of sentence $\mathbf{s}_{j}$ in the first term can be decomposed as

$$
\begin{aligned}
\mathcal{L}^{s_{j}}(\beta, \pi ; \xi, \gamma)= & \mathbb{E}_{q}\left[\log p\left(\epsilon^{j} \mid \pi\right)\right]-\mathbb{E}_{q}[\log q(\mathbf{z})]+\sum_{n=1}^{N_{j}} \mathbb{E}_{q}\left[\log p\left(z_{n} \mid \epsilon^{j}, \theta^{j-C: j-1}, \theta^{j+1: j+C}, \vartheta^{d}\right)\right] \\
& +\sum_{n=1}^{N_{j}} \mathbb{E}_{q}\left[\log p\left(w_{n} \mid z_{n}, \beta\right)\right]-\mathbb{E}_{q}\left[\log q\left(\epsilon^{j}\right)\right] .
\end{aligned}
$$

Here $\theta^{j-C: j-1}$ and $\theta^{j+1: j+C}$ denote the topic matrices of the preceding sentences and the subsequent sentences for the sentence $\mathbf{s}_{j}$, respectively. Note that, in order to show the inference more intuitive, we introduce the form of an augmented matrix to the term $\mathbb{E}_{q}\left[\log p\left(z_{n} \mid \epsilon^{j}, \theta^{j-C: j-1}, \theta^{j+1: j+C}, \vartheta^{d}\right)\right]$ as

$$
\sum_{n=1}^{N_{j}} \mathbb{E}_{q}\left[\log p\left(z_{n} \mid \epsilon^{j}, \theta^{j-C: j-1}, \theta^{j+1: j+C}, \vartheta^{d}\right)\right]=\sum_{n=1}^{N_{j}} \mathbb{E}_{q}\left[\log p\left(z_{n} \mid \epsilon^{j},\left(\theta^{j-C: j-1} / \theta^{j+1: j+C} / \vartheta^{d}\right)\right)\right],
$$

where $\left(\theta^{j-C: j-1} / \theta^{j+1: j+C} / \vartheta^{d}\right)$ is the augmented matrix of $\theta^{j-C: j-1}, \theta^{j+1: j+C}$ and $\vartheta^{d}$ by merging the three matrices into a matrix. We use $\theta^{2 C+1}$, which is a $(2 C+1) \times K$ matrix, to denote this augmented matrix.

Thus, we can use an alternating optimization to maximize the lower-bound $\mathcal{L}^{\mathrm{d}}\left(\beta, \pi, \vartheta^{d} ; \xi, \gamma, \rho^{d}\right)$ to learn the model parameters, i.e., $\beta$ and $\pi$. We present a variational expectation-maximization (EM) algorithm for the inference. In the E-step, we first need to maximize $\mathcal{L}^{s_{j}}(\beta, \pi ; \xi, \gamma)$ to find the estimations of the variational parameters $(\xi, \gamma)$ with $\vartheta^{d}$ fixed. After scanning the two directional sequences of the sentences in a document $d$, we maximize $\mathcal{L}^{\mathrm{d}}\left(\beta, \pi, \vartheta^{d} ; \xi, \gamma, \rho^{d}\right)$ with respect to $\vartheta^{d}$ 
and $\rho^{d}$ in the M-step, which is shown in the following sections. The detailed formulations about the optimization of Bi-RATM are in the Appendix.

\subsection{Variational Update for Attention Signals}

Based on $\mathcal{L}^{s_{j}}(\beta, \pi ; \xi, \gamma)$, for the variational parameters $\xi$ corresponding to the attention signals of sentence $s_{j}$, we need to maximize the following equation as

$$
\begin{aligned}
\mathcal{L}_{[\xi]}^{s_{j}}= & \sum_{l=1}^{2 C+1}\left(\pi_{l}-1\right)\left(\Psi\left(\xi_{l}\right)-\Psi\left(\sum_{l^{\prime}=1}^{2 C+1} \xi_{l^{\prime}}\right)\right)-\log \Gamma\left(\sum_{l=1}^{2 C+1} \xi_{l}\right)+\sum_{l=1}^{2 C+1} \log \Gamma\left(\xi_{l}\right) \\
& -\sum_{l=1}^{2 C+1}\left(\xi_{l}-1\right)\left(\Psi\left(\xi_{l}\right)-\Psi\left(\sum_{l^{\prime}=1}^{2 C+1} \xi_{l^{\prime}}\right)\right)+\sum_{n=1}^{N_{j}} \sum_{k=1}^{K} \gamma_{n k} \sum_{l=1}^{2 C+1} \log \theta_{l k}^{2 C+1} \frac{\xi_{l}}{\sum_{l^{\prime}=1}^{2 C+1} \xi_{l^{\prime}}},
\end{aligned}
$$

where $\Psi(\cdot)$ is the digamma function, which is the first derivative of the logarithm of the Gamma function. Here we use the gradient descent method to estimate $\xi$.

After learning the attention signals for the sentence $\boldsymbol{s}_{j}$, we can update $\theta^{j}$, the topic distribution of the $\mathbf{s}_{j}$, as

$$
\theta_{k}^{j}=\sum_{l=1}^{2 C+1} \frac{\xi_{l}}{\sum_{l^{\prime}} \xi_{l^{\prime}}} \theta_{l k}^{2 C+1}
$$

which is the basic assumption of the proposed model that the topic distribution of the $j$-th sentence is obtained by its preceding sentences and subsequent sentences with the corresponding attentional signals. Note that we initialize the topic distribution of all the sentences in $\mathbf{d}^{i}$ by the topic distribution of $\mathbf{d}^{i}$ at the beginning of model learning.

5.1.1 Variational Update for Word Assignment. For each word $w_{n}$ in sentence $s_{j}$, a topic index $z_{n}$ is assigned to $w_{n}$ and $\gamma_{n k}$ is the variational parameter corresponding to the probability that the topic $k$ is assigned to the word $w_{n}$. The update equation of $\gamma_{n k}$ is as follows:

$$
\gamma_{n k} \propto \beta_{k, v^{w_{n}}} \exp \sum_{l=1}^{2 C+1} \log \theta_{l k}^{2 C+1} \frac{\xi_{l}}{\sum_{l^{\prime}} \xi_{l^{\prime}}},
$$

where $v^{w_{n}}$ denotes the index of the word $w_{n}$ in the dictionary.

The traditional topic models based on the bag-of-words assumption would stumble when the document is too short, which has been discussed in some previous works [66]. The proposed model is capable of handling short documents because it fully utilizes the topic information from the preceding sentences and the subsequent sentences (See the summand in Equation (7)) with adaptive attention signals to generate the topic distribution for the current sentence.

\subsection{Variational Update for Document Embedding}

As one kind of topic models, the main purpose of bi-RATM is to extract the topic distribution of each document for document embedding. In the above variational inference, we define $G_{0}=\delta_{\vartheta^{d}}$ and update the topic distribution for the whole document, $\vartheta^{d}$, which is the embedding of one document. Note that we treat $\epsilon_{2 C+1}$ as the attention signal for $\vartheta^{d}$ as described in the bi-RABP. When $\{\beta, \pi, \xi, \gamma\}$ are fixed in the alternating optimization, we maximize $\mathcal{L}^{\mathbf{d}}\left(\beta, \pi, \vartheta^{d} ; \xi, \gamma, \rho^{\mathbf{d}^{i}}\right)$ with respect to $\vartheta^{d}$ and $\rho^{\mathrm{d}^{i}}$. By setting the derivative with respect to $\rho^{\mathrm{d}^{i}}$ to 0 , we can obtain an analytical solution as

$$
\rho_{k}^{\mathrm{d}^{i}}=\alpha_{k}+\sum_{j=1}^{S_{i}} \sum_{n=1}^{N_{j}} \gamma_{n k} \cdot \frac{\xi_{C}}{\sum_{l}^{C} \xi_{l}},
$$

where $\alpha$ is initialized by LDA and then fixed as LDA did. 
With $\rho_{k}^{\mathrm{d}^{i}}$, we can obtain $\vartheta^{d^{i}}$ as a normalized $\left\{\rho_{k}^{\mathrm{d}^{i}}\right\}$ according to [7] by

$$
\vartheta_{k}^{d^{i}}=\frac{\rho_{k}^{\mathrm{d}^{i}}}{\sum_{k^{\prime}} \rho_{k^{\prime}}^{\mathrm{d}^{i}}}
$$

\subsection{Parameter Estimation}

The model parameters include $\pi$ and $\beta$. In the M-step, we can estimate the parameters by maximizing a lower bound of the log-likelihood based on the variational E-step.

Based on $\mathcal{L}^{\mathrm{d}}\left(\beta, \pi, \vartheta^{d} ; \xi, \gamma, \rho^{d}\right)$, the objective function for $\pi$ to be maximized over the whole corpus is formulated as

$$
\mathcal{L}_{[\pi]}^{d}=\sum_{i=1}^{M} \sum_{j=1}^{S_{i}}\left(\log \Gamma\left(\sum_{l=1}^{2 C+1} \pi_{l}\right)-\sum_{l^{\prime}=1}^{2 C+1} \log \Gamma\left(\pi_{l^{\prime}}\right)+\sum_{l=1}^{2 C+1}\left(\pi_{l}-1\right)\left(\Psi\left(\xi_{l}\right)-\Psi\left(\sum_{l^{\prime}=1}^{2 C+1} \xi_{l^{\prime}}\right)\right)\right) .
$$

We use the linear-time Newton-Raphson algorithm described in the LDA to estimate $\pi$.

The update equation of $\beta_{k v}$ is,

$$
\beta_{k v}=\sum_{i=1}^{M} \sum_{j=1}^{S_{i}} \sum_{n=1}^{N_{j}} \gamma_{n k} \cdot w_{n}^{v}
$$

where $v$ is the index of $w_{n}$ in the dictionary.

The optimization procedure iterates until convergence. We show the detailed variational EM procedure in Algorithm 1.

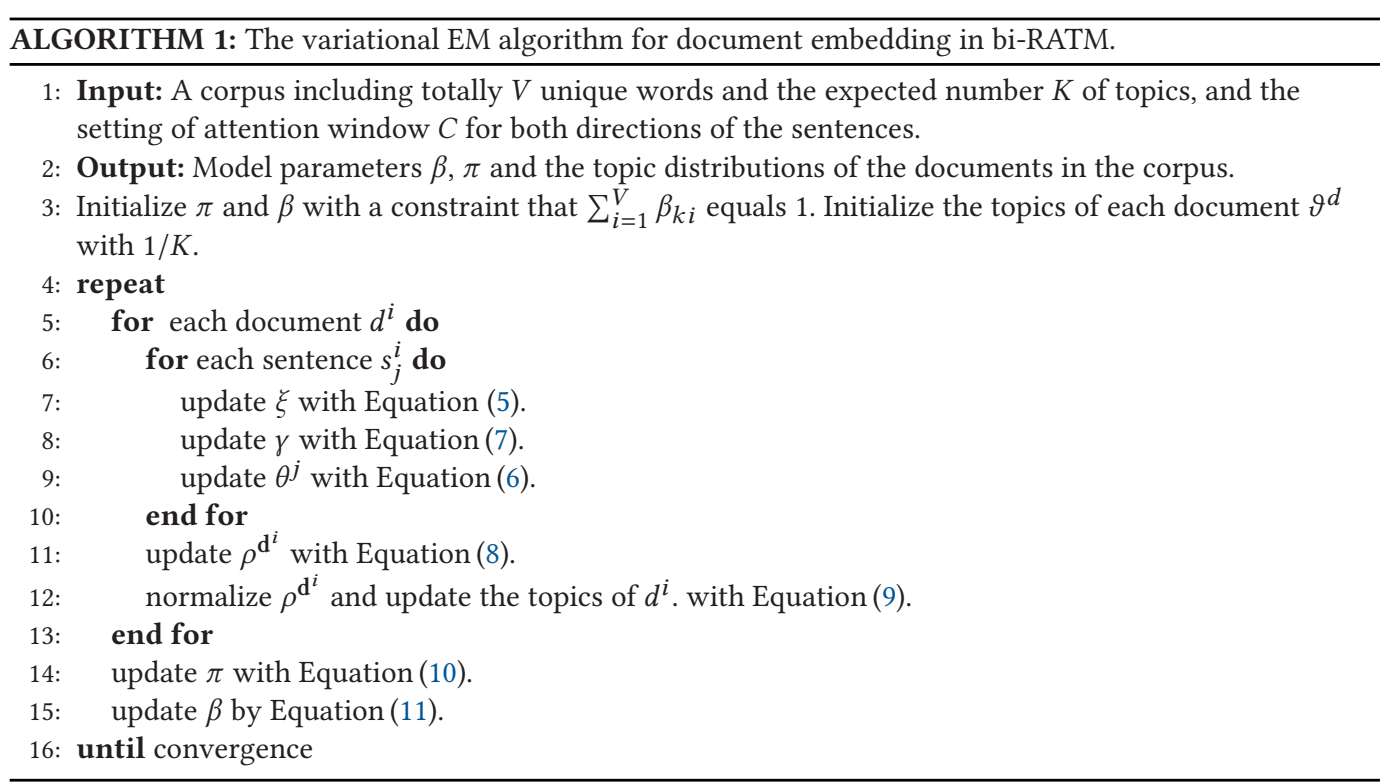

\section{ONLINE BI-RATM}

Algorithm 1 describes the variational expectation maximization procedure for bi-RATM. This algorithm is a batch algorithm and requires a full pass through the entire corpus in each iteration. Thus, it can be very slow when handling very large datasets $[8,50]$. Moreover, where new data is constantly arriving, it can only learn from scratch with high complexity. To deal with large-scale 
document stream, we propose an online variational Bayesian inference for bi-RATM, namely, online bi-RATM. A common technique in online stochastic learning is to consider mini-batches of data per update to reduce noises $[8,39]$. Thus, we treat the large-scale document stream as a sequential mini-batch $b(b \in(0, \infty))$ as shown in [30].

There are three different variational parameters in bi-RATM, namely, $\xi, \gamma$ and $\rho$, which are document-specific. Thus, for the online variational Bayesian algorithm, our goal is to learn model parameters $\beta$ and $\pi$ by maximizing the $\operatorname{ELBO} \mathcal{L}(\cdot)$ after updating the variational parameters $\xi, \gamma$, and $\rho$ in the E-step of Algorithm 1 .

We define $b$ to denote a new mini-batch in a document stream, where a mini-batch $b$ contains $B$ documents. When a new mini-batch $b$ is observed, we perform an E-step to update $\xi, \gamma$ and $\rho$ for each document in $b$. After that, we need to update $\beta$ and $\pi$ based on the batch $b$. We describe this process in two steps.

First, we update $\beta$, the topic distributions over words, with the new batch $b$. We let $\beta_{b}$ denote the component of $\beta$, which is learned after we run through the bi-RATM over the new batch $b$. We need to update $\beta$ based on $\beta_{b}$. In order for this, we compute $\nabla \beta$, the gradient of $\beta$ with respect to $\beta_{b}$. We update $\beta$ using a weighted average of its previous values and $\nabla \beta$. As described in $[12,30$, 57], the weight given to $\beta_{b}$ is defined as

$$
\sigma^{b}=\left(\tau_{0}+b\right)^{-k}, \tau_{0} \geq 0
$$

where $k \in(0.5,1]$ controls the rate at which old values of $\beta_{b}$ are forgotten. Based on a new batch $b$, we compute the gradient of $\beta$ as

$$
\nabla \beta=\beta-\beta_{b} .
$$

Then, we update $\beta$ by a stochastic natural gradient algorithm as

$$
\beta=\beta-\sigma^{b} \cdot \nabla \beta=\beta-\sigma^{b} \cdot\left(\beta-\beta_{b}\right)=\left(1-\sigma^{b}\right) \beta+\sigma^{b} \beta_{b} .
$$

Second, we can update $\pi$ as the same way as $\beta$. The updating rule is formulated as

$$
\pi=\left(1-\sigma^{b}\right) \pi+\sigma^{b} \pi_{b}
$$

The detailed algorithm for the online bi-RATM is shown in Algorithm 2.

\section{MODEL ANALYSIS}

In this section, we discuss the difference between the proposed bi-RATM and the conventional LDA. Then we show the connection between bi-RATM and matrix factorization techniques.

\subsection{Comparison with the Conventional LDA}

When we let $C=0$, Equation (2) will become

$$
\theta^{t} \mid \theta^{t-C: t-1}, \theta^{t+1: t+C}, G_{0}, \pi \sim \epsilon_{2 C+1} \cdot G_{0},
$$

where $\epsilon_{2 C+1}=1$ means that the topic distribution of current $\theta^{t}$ follows the base distribution $G_{0}$. Thus, with $G_{0}=\delta_{\vartheta^{d}}$ and $C=0$ in bi-RATM, the variational parameter $\rho_{k}^{\mathrm{d}^{i}}$ will be

$$
\rho_{k}^{\mathrm{d}^{i}}=\alpha_{k}+\sum_{j=1}^{S_{i}} \sum_{n=1}^{N_{j}} \gamma_{n k},
$$

where $\gamma_{n k}$ is the probability assignment for a word in a sentence. This equation is the same as that of the variational topic distribution in LDA. 


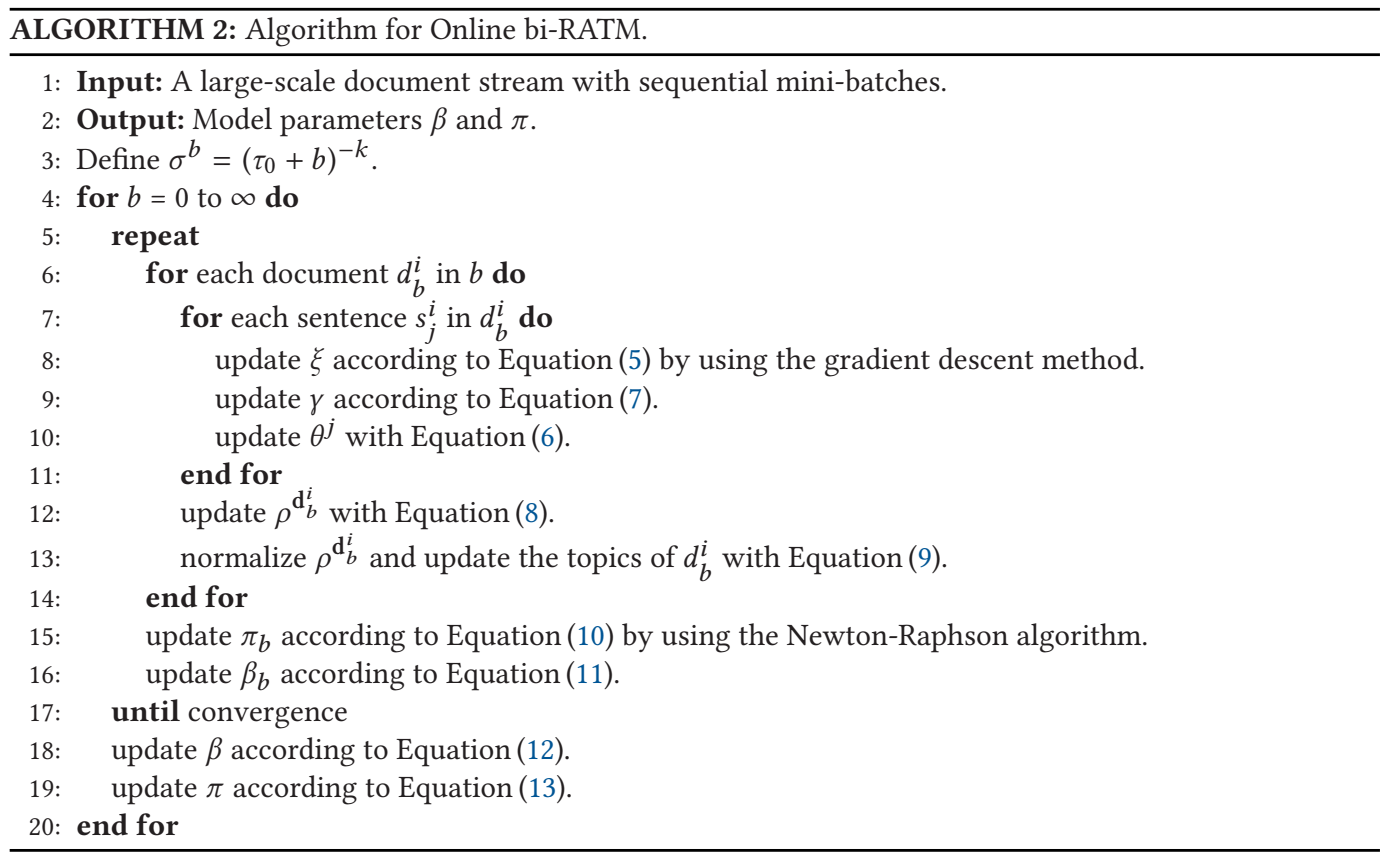

Equation (7) describes the word assignment with the variational parameter $\gamma_{n k}$. Taking the base distribution into consideration and letting $C=0$, we can rewrite the word assignment function as

$$
\gamma_{n k} \propto \beta_{k, v^{w_{n}}} \exp \left\{\mathbb{E}_{q}\left[\log p\left(\vartheta^{d} \mid \alpha\right)\right]\right\},
$$

which is exactly the same as the variational update for the word assignment described in LDA. Thus, it is interesting to note that bi-RATM degenerates into LDA without attention signals. Similarly, if we use a uniform $\epsilon$, the bi-RATM will also degenerate to the standard LDA.

\subsection{Connection to Matrix Factorization}

Non-negative matrix factorization (NMF) on topic modeling is proved to be equivalent to optimizing the objective function of the Probabilistic Latent Semantic Analysis (PLSA) [20]. It is well known that PLSA is simply a special case of LDA. In this section, we will analyze the bi-RATM from the perspective of matrix factorization and show the connection between bi-RATM and LDA.

Consider a corpus with $M$ documents by $D=\left\{\mathbf{d}^{1}, \ldots, \mathbf{d}^{M}\right\}$, where $\mathbf{d}^{i}(i \in\{1, \ldots, M\})$ denotes the $i$-th document. The dictionary contains $V$ words from the corpus. Let $F=\left(F_{i j}\right)$ be the documentword matrix, where $F_{i j}=F\left(d_{i}, w_{j}\right)$ denotes the frequency of word $w_{j}$ in document $d_{i}$.

General speaking, topic modeling tries to factorize the matrix $F$ into two different nonnegative matrices $U$ and $H$ with a fixed $K$, where $K$ denotes the number of topics. $U$, an $M \times K$ matrix, can be treated as the matrix of document distribution over latent topics, and $H$, a $V \times K$ matrix, denotes the matrix of the topic distributions over the dictionary. Thus, from the perspective of matrix factorization, PLSA and LDA can be unified as:

$$
F=U H^{\mathrm{T}} .
$$

In bi-RATM, each sentence is represented as a basic unit under the bag-of-words assumption. As above, we take $s_{j}^{i}, j \in\left\{1, \ldots, S_{i}\right\}$, to denote the $j$-th sentence in $\mathrm{d}^{i}$ and $\mathrm{s}_{j}^{i}=\left(w_{j, 1}^{i}, \ldots, w_{j, N_{j}^{i}}^{i}\right)$ to denote the vector of $N_{j}^{i}$ words associated with sentence $s_{j}^{i}$. bi-RATM defines the distribution 
of each sentence over the latent topics, $\theta^{s}$, as shown above. Let $U^{s}$, a $S_{\text {all }} \times K$ matrix, denote the matrix of all the $\theta^{s}$ 's, where $S_{\text {all }}$ is the total number of sentences in the whole corpus. The attentional signals in bi-RATM can also be constructed via an $S_{\text {all }} \times S_{\text {all }}$ matrix $A$. Each entry $A_{i j}$ in $A$ indicates the neighborhood relationship between the sentence $s^{i}$ and the sentence $\mathbf{s}^{j}$ and the

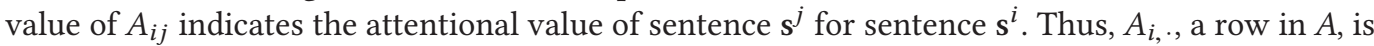
the attention vector for the sentence $s^{i}$, where only the elements corresponding to the neighbored sentences of $\mathbf{s}^{i}$ could be nonzero. Note that the number of nonzero entries in each row of $A$ equals $2 C$, because we consider both of the preceding and subsequent sentences of sentence $s^{i}$. Hence, $A$ could be very sparse because the number of sentences in the corpus could be very large. Let $E$ denote an $M \times S_{\text {all }}$ binary matrix to model the relationship between documents and sentences, where $E_{i j}$ is set to 1 if sentence $\mathbf{s}^{j}$ is in the document $\mathbf{d}^{i}$ and 0 otherwise. Then the bi-RATM can be understood from the perspective of matrix factorization as:

$$
F=E A U^{s} H^{T} .
$$

It is worth to note that, if only the preceding sentences are considered, which is the basic RATM described in our earlier work [38], we can also obtain the same results from the above analysis, where nonzero entries correspond to the preceding sentences of sentence $\mathbf{s}^{i}$. Thus, compared with LDA, bi-RATM takes advantage of the bi-directional sequential information of sentences and the corresponding attention signals.

We can set all the non-zero elements in the attention matrix $A$ to 1 , which means that all the attentions are equal, and all of the neighbored sentences have the same effect on the generation of the topics for the current sentence. Here we set $A_{i i}=1$ for the sentences that have no neighbored sentence. Under this setting, the result of $E A U^{s}$ will be a $M \times K$ matrix, which is named as $U^{\prime}$. We find that the $i$-th row of $U^{\prime}$ can be formulated as

$$
U_{i}^{\prime}=\left(S_{i} \sum_{j=1}^{S_{i}} U_{j 1}^{s}, S_{i} \sum_{j=1}^{S_{i}} U_{j 2}^{s}, \ldots, S_{i} \sum_{j=1}^{S_{i}} U_{j k}^{s}\right) \propto\left(\sum_{j=1}^{S_{i}} U_{j 1}^{s}, \sum_{j=1}^{S_{i}} U_{j 2}^{s}, \ldots, \sum_{j=1}^{S_{i}} U_{j k}^{s}\right),
$$

where $S_{i}$ represents the number of the sentences in $\mathbf{d}^{i}$. It is easy to see that Equation (15) can be simplified to $F=U^{\prime} H^{T}$, which is similar to Equation (14) at the sentence level. Differently, the topic distribution of a document in $U^{\prime}$ is computed by averaging the topics of all the sentences in the document. Thus, we find that bi-RATM works by decomposing the documents into the sentence level.

\section{COMPLEXITY ANALYSIS}

In this section, we discuss the time complexity of the variational inference for the proposed biRATM by comparing with the traditional mean-field variational inference of the LDA. For each iteration of the LDA, the mean-field variational inference needs to compute the variational parameters over the $K$ topics through the corpus. The time complexity of the LDA is $O\left(M \times N_{d} \times K\right)$, where $M$ denotes the number of documents in the corpus, and $N_{d}$ is the number of words in a document. Recall that $S_{i}$ is the number of sentences in $\mathrm{d}^{i}$ and $N_{j}^{i}$ is the number of words in $\mathbf{s}_{j}^{i}$. With the variational EM algorithm of bi-RATM shown in Algorithm 1, the time complexity of the bi-RATM is $O\left(M \times S_{i} \times N_{j}^{i} \times(2 C+1) \times K+M \times S_{i} \times N_{j}^{i} \times K\right)$ per-iteration. Since $N_{d} \approx S_{i} \times N_{j}^{i}$, the time complexity of the bi-RATM is approximately $O\left(M \times N_{d} \times K \times(2 C+2)\right)$.

According to experimental results shown in Section 9, $C$ is small in many scenarios. Meanwhile, $M \ggg N_{d}$ and $M \ggg K$; thus, the time complexity of the proposed model highly depends on the scale of the corpus. The proposed online algorithm in Algorithm 2 runs through a small batch of 
the corpus, where the batch size $B$ is much smaller than $M$. Hence it would greatly reduce the complexity of the bi-RATM method in many real applications.

\section{EXPERIMENTS}

In this section, we empirically evaluate the proposed model on document modeling, topic coherence and classification tasks. We also test the performance of the proposed online algorithm on a large corpus. The source codes and data are released to the public. ${ }^{1}$

\subsection{Datasets}

The proposed bi-RATM model is evaluated on two corpora. The first corpus is a subset of the Wikipedia. We extract the abstract of each page in Wikipedia and remove abstracts with less than 5 sentences to form the corpus, which contains 241,290 documents. We remove stop words and obtain a vocabulary of 21,968 words. Each document belongs to 1 of 68 categories such as education, book, and arts, and the average number of sentences in this corpus is about 7 . The second corpus we used is news articles from New York Times (NYTimes) from January 1st, 2016 to May 8th, 2016. After removing articles which contain less than 5 sentences, we obtain 27,523 articles, each of which belongs to one of 42 categories such as world, movies, sports, and magazine. After removing stop words, we obtain a dictionary with 12,047 words and the average number of sentences in the NYTimes corpus is about 40.

To test online bi-RATM, we experiment on a larger dataset from Wikipedia, which contains 906,117 pages. We use the top 10,000 words appeared in the whole dataset as the dictionary. We split this dataset into 453 batches, where the average number of pages in each batch is 2,000 .

\subsection{Experiments on Document Modeling}

In this part of the experiments, we show the performance of the proposed model on document modeling.

We consider the following baselines.

(1) LDA [7]. We use the variational inference implementation of LDA from the standard toolkit. ${ }^{2}$

(2) CTM [6]. We use the implementation from Blei's toolkit with default hyperparameters. ${ }^{3}$

(3) Hierarchical Dirichlet processes (HDP) [67]. HDP handle the corpus without considering the initial topic numbers, where the data determine the number of topics. We use the implementation from Blei's toolkit with the default parameters. ${ }^{4}$

(4) RSM [29]: RSM models the documents through a two-layer neural network, which can be used to model and automatically extract low-dimensional latent semantic representations from a large unstructured collection of documents.

(5) HTMM [27]. HTMM is proposed to model the topics of the words in a document as a Markov chain, which assumes that the subsequent words are more likely to have the same topic. The reason we choose HTMM as a baseline is that HTMM is proposed to model the sequential topics of words. We use the public implementation for HTMM. ${ }^{5}$

(6) Gaussian Mixture Neural Topic Model (GMNTM) [75]. GMNTM is a Gaussian mixture model which jointly learns the topic model and the vector representation. This work also models the word sequences where the word sequential information is used to model the semantics of the document, which is same as Gruber's work [27].

\footnotetext{
$\overline{{ }^{1} \text { http://www.shuangyin.li/biratm/. }}$

${ }^{2}$ http://www.cs.columbia.edu/ blei/lda-c/index.html.

${ }^{3}$ http://www.cs.columbia.edu/ blei/ctm-c/index.html.

${ }^{4}$ http://www.cs.columbia.edu/ blei/topicmodeling_software.html.

${ }^{5}$ http://www.cs.toronto.edu/ amitg/.
} 
Table 1. The Held-out Perplexity of Different Models on the Two Corpora When $K=50,100,200,400,600$

\begin{tabular}{|c|c|c|c|c|c|c|c|c|c|c|}
\hline \multirow{2}{*}{ Models } & \multicolumn{9}{|c|}{ Wikipedia } & \multicolumn{6}{c|}{ NYTimes } \\
\cline { 2 - 11 } & $K=50$ & $K=100$ & $K=200$ & $K=400$ & $K=600$ & $K=50$ & $K=100$ & $K=200$ & $K=400$ & $K=600$ \\
\hline LDA & 585.08 & 493.73 & 402.98 & 286.09 & 226.76 & 794.81 & 768.24 & 748.45 & 489.20 & 435.66 \\
CTM & 524.11 & 435.67 & 440.89 & 893.08 & 992.73 & 730.60 & 645.41 & 736.38 & 788.01 & 1230.64 \\
HDP & 728.03 & 728.03 & 728.03 & 728.03 & 728.03 & 1582.67 & 1582.67 & 1582.67 & 1582.67 & 1582.67 \\
RSM & 752.36 & 750.08 & 767.08 & 741.06 & 731.30 & 1259.10 & 1251.71 & 1266.5 & 1051.84 & 992.77 \\
HTMM & 565.34 & 468.30 & 382.23 & 271.40 & 215.81 & 753.88 & 738.67 & 719.90 & 564.01 & 513.22 \\
GMNTM & 550.12 & 433.32 & 359.04 & 254.92 & 206.09 & 700.34 & 677.13 & 656.84 & 435.36 & 382.59 \\
SeqLDA & 536.07 & 444.08 & 385.88 & 254.15 & 214.96 & 870.50 & 687.15 & 721.06 & 436.62 & 412.97 \\
RATM & 553.87 & 402.47 & 328.6 & 162.90 & 147.74 & 576.06 & 493.44 & 500.07 & 400.12 & 368.24 \\
RATM-D & 532.72 & 392.05 & 314.47 & 150.98 & 127.02 & 529.08 & 442.65 & 440.68 & 386.62 & 335.68 \\
bi-RATM & $\mathbf{4 1 4 . 0 1}$ & $\mathbf{2 9 1 . 6 0}$ & 200.43 & $\mathbf{1 2 7 . 5 5}$ & $\mathbf{1 1 8 . 8 4}$ & $\mathbf{5 0 1 . 3 3}$ & $\mathbf{4 1 1 . 3 2}$ & 369.54 & $\mathbf{3 0 0 . 1 9}$ & $2 \mathbf{2 9 0 . 1 9}$ \\
\hline
\end{tabular}

(7) SeqLDA [21]. SeqLDA considers the underlying sequential structure, i.e., a document consisting of multiple segments such as chapters and paragraphs, where each segment is correlated to its preceding and subsequent segments. In experiments, we treat sentences as the segments of a document in SeqLDA.

Besides, we also compare with RATM model [38] that is a special case of bi-RATM. The standard RATM is trained without using $G_{0}$ for the generation of each sentence and hence it does not update $\vartheta^{d}$ for each document as well as the responding variational parameters $\rho^{d^{i}}$. Moreover, one variant of RATM named as RATM-D is considered as a baseline. The RATM-D just uses the topic distribution of the host document as the $G_{0}$. In the RATM and RATM-D, the first $C-1$ sentences in a document do not have $C-1$ preceding sentences, which brings difficulties to the use of the attention signals in the RABP. In this case, we just use the topic distribution of the host document, $\vartheta^{d}$, instead. We set different window sizes for the Wikipedia and NYTimes corpus when we train the RATM and RATM-D. For the Wikipedia corpus, $C$ is set to be 4 , and for the NYTimes corpus, $C$ is 6 to get the best results. For the proposed bi-RATM model, $C$ is set to be 3 for the Wikipedia corpus, and $C$ is 6 for the NYTimes corpus, respectively.

To compare the performance of different models, we use the held-out perplexity as a measure, which is defined for topic models on the sentence level as

$$
\operatorname{perplexity}\left(D_{\text {test }}\right)=\exp \left(-\frac{\sum_{i=1}^{M} \sum_{j=1}^{S_{i}} \log p\left(\mathrm{~s}_{j}^{i}\right)}{\sum_{i=1}^{M} \sum_{j=1}^{S_{i}} N_{j}^{i}}\right),
$$

where $M$ denotes the number of documents in the test set and $\sum_{j=1}^{S_{i}} N_{j}^{i}$ is the total number of words in document $d^{i}$. The lower the perplexity is, the better the performance is.

In each corpus, $80 \%$ of documents are used for training and the rest is for testing. That is, for the Wikipedia corpus, there are 200,000 documents for training and 41,290 documents for testing. For the NYTimes corpus, 22,000 documents are used for training and 5,523 documents for testing.

To see the effect of the number of latent topics $K$, Table 1 shows the held-out perplexities of different methods on the Wikipedia and NYTimes corpora when the number of latent topics takes 5 values, i.e., 50, 100, 200, 400, and 600. HDP holds the same results when $K$ increases because HDP does not need to assign the topic number before learning. The topic number learned by HDP is $K=713$ for Wikipedia and $K=676$ for NYTimes, respectively.

The results show that the RATM and RATM-D have much better performance than baseline models. The performance of the bi-RATM is better than that of the RATM and RATM-D, which 
demonstrates that the incorporation of the bi-directional sequences of the whole document can bring benefits for performance improvement. Moreover, when the number of topics increases, the performance of the bi-RATM tends to become better due to the increased capacity.

Moreover, bi-RATM takes advantage of more information than both the RATM and RATM-D, when we model the sentences of a document based on the assumption of bi-directional sequences. That is, in RATM and RATM-D, only the $C$ preceding sentences are considered for a sentence in the Wikipedia corpus, while the $C$ preceding sentences and $C$ subsequent sentences are considered together in bi-RATM.

\subsection{Analysis on Topic Coherence}

In this section, we use the topic coherence score to show the effects of the scale of the corpus and the number of topics, where the topic coherence score is always used to measure how well the topics are extracted [69].

The topic coherence $[47,49,63]$ scores a single topic by measuring the degree of the semantic similarity between high scoring words in the topic. This measurement helps distinguish between topics that are semantically interpretable artifacts of statistical inference. In the experiments, we take advantages of two coherence measures, both of which have been shown to match well with human judgements of the topic quality.

(1) The UCI measure [49], an extrinsic measure, defines the score of a pair of words to be the Point-wise Mutual Information between those two words. The UCI measure for a topic $k$ is defined as

$$
\operatorname{UCI}\left(w_{i}, w_{j}\right)_{k}=\log \frac{p\left(w_{i}, w_{j}\right)}{p\left(w_{i}\right) p\left(w_{j}\right)},
$$

where $p(w)$ represents the probability of $w_{i}$ in a document, and $p\left(w_{i}, w_{j}\right)$ is the probability of both $w_{i}$ and $w_{j}$ co-occurring in the same document. Those probabilities can be computed from the test set $D_{\text {test }}$ by counting the occurrences, e.g., $p\left(w_{i}\right)=\#\left(w_{i}\right) / \#\left(D_{\text {test }}\right)$. Thus, the larger the UCI score is, the better the topic is.

(2) The UMass measure [47], an intrinsic measure, is defined for a topic $k$ as

$$
\operatorname{UMass}\left(w_{i}, w_{j}\right)_{k}=\log \frac{D\left(w_{i}, w_{j}\right)+1}{D\left(w_{i}\right)},
$$

where $D\left(w_{i}\right)$ denotes the counts of $w_{i}$ in the test set $D_{\text {test }}$. Similarly, the larger the UMass score is, the better the topic is. In practice, we rank words in a topic based on their probabilities and then select top words to compute the UCI and UMass scores as top words are more representative for that topic.

To see the effect of the scale of the corpus, we sample $D=5 k, 10 k, 20 k, 40 k, 80 k$, and $120 k$ from Wikipedia to train LDA, CTM, RATM-D, and bi-RATM, respectively. We compute the UCI and UMass scores with $K=100$, and top 20 words in each topic are considered. Since the topic coherence is evaluated for a single topic, we first compute the topic coherence for each topic, and then show the average coherence of all topics. Table 2 shows the experimental results. From Table 2, we observe that when the training data is small, LDA performs better, while the performance of bi-RATM becomes better when the size of the training set increases. That is because that bi-RATM is more complicated than LDA and hence it needs more data to train.

To see the effect of the number of topics, we compute the UCI and UMass scores with $K=50$, $100,200,400$, and 600 by training LDA and bi-RATM on the Wikipedia corpus with $D=120 \mathrm{k}$. As shown in Section 9.2, the topic number learned by HDP on Wikipedia is $K=713$. We also report the UCI and UMass scores of LDA and bi-RATM with the topic number learned by HDP. In this part of the experiments, we set $C=3$ when bi-RATM is trained, which is the best setting of $C$ for 
Table 2. The UCI and UMass Scores with Topic $K=100$ on Different Data Sizes

\begin{tabular}{|c|c|c|c|c|c|c|c|c|c|c|c|c|}
\hline & \multicolumn{9}{|c|}{ UCI } & \multicolumn{1}{c|}{ UMass } \\
\cline { 2 - 12 } & $5 k$ & $10 k$ & $20 k$ & $40 k$ & $80 k$ & $120 k$ & $5 k$ & $10 k$ & $20 k$ & $40 k$ & $80 k$ & $120 k$ \\
\hline LDA & 113.59 & 170.41 & 206.17 & 225.51 & 235.42 & 236.91 & -984.65 & -974.12 & -965.12 & -938.56 & -951.07 & -953.25 \\
CTM & 139.03 & 131.37 & 108.56 & 91.67 & 70.03 & 30.04 & -941.31 & -980.82 & -1025.62 & -933.70 & -950.93 & -814.98 \\
RATM-D & 100.93 & 166.29 & 205.53 & 215.50 & 221.62 & 223.21 & -1100.35 & -1038.92 & -988.54 & -968.94 & -955.42 & -952.94 \\
bi-RATM & 109.30 & 164.50 & 203.72 & 224.79 & 228.38 & 231.00 & -1056.45 & -994.17 & -962.53 & -930.72 & -943.82 & -938.26 \\
\hline
\end{tabular}

Table 3. The UCI and UMass Scores with Topic $K=50,100,200,400,600,713$

\begin{tabular}{|c|c|c|c|c|c|c|c|c|c|c|c|c|}
\hline & \multicolumn{9}{|c|}{ UCI } & \multicolumn{6}{c|}{ UMass } \\
\cline { 2 - 12 } & $K=50$ & $K=100$ & $K=200$ & $K=400$ & $K=600$ & $K=713$ & $K=50$ & $K=100$ & $K=200$ & $K=400$ & $K=600$ & $K=713$ \\
\hline LDA & 204.80 & 236.91 & 246.77 & 239.26 & 218.21 & 212.55 & -908.66 & -953.25 & -1001.50 & -1037.28 & -1022.94 & -1025.76 \\
bi-RATM & 203.17 & 231.00 & 148.54 & 244.50 & 253.98 & 254.19 & -895.13 & -938.26 & -1040.59 & -1002.14 & -988.65 & -991.35 \\
\hline
\end{tabular}

Table 4. The Held-out Perplexities of bi-RATM on Wikipedia with $K=100$ when Changing $C$

\begin{tabular}{|c|c|c|c|c|c|}
\hline$\# / K=100$ & $C=2$ & $C=3$ & $C=4$ & $C=5$ & $C=6$ \\
\hline bi-RATM & 320.92 & 291.60 & 301.03 & 338.74 & 350.01 \\
\hline
\end{tabular}

Wikipedia. Similarly, the top 20 words in each topic are considered and the average coherence of all topics is used as a measure. Table 3 shows the experimental results. From Table 3, we observe that LDA overfits when $K=600$ as it has a low UCI score. The UCI score of bi-RATM increases with the number of topics, even though bi-RATM gets low UCI and UMass scores when $K=200$. The bi-RATM achieves the best result on the UCI score when $K=713$, while, it is nearly close to the result of $K=600$, which means that for Wikipedia, we can set the best $K$ in a range $(600,713)$ in consideration of the model complexity. On the other hand, this suggests that we could run the HDP model to learn a suitable $\mathrm{K}$ for a corpus before bi-RATM is trained, which is a common and effective approach for topic models in practice.

\subsection{Analysis on Attention Signals}

In the section, we show the effect of the attention signals learned in the bi-RATM model. The attention signals indicate the importance of the preceding and subsequent sentences to the current one in a document. We will test our model in three different perspectives.

9.4.1 Analysis on $C$. First, we study the effect of $C$ on the performance of bi-RATM. We set $C=2,3,4,5,6$ on the Wikipedia corpus with $K=100$. As shown in Table 4, we find that bi-RATM achieves the best result on $C=3$. The performance becomes worse when $C$ is larger than 3 . This suggests that choosing a small value for $C$ can achieve good performance on the Wikipedia corpus. For the NYTimes corpus, bi-RATM performs the best on $C=6$ as RATM and RATM-D did.

9.4.2 Analysis on Attention Signals. Second, to show the values of the attention signals, we set $C$ to be 3 and train the RATM, RATM-D, and bi-RATM on the Wikipedia corpus with the same settings, respectively. We choose the topic distribution of the host document as $G_{0}$. Table 6 shows the values of attention signals for some documents in the Wikipedia corpus (See Table 5) and the numbers are the values of attention signals of the preceding sentences and the host document 
Table 5. The Cases from Wikipedia $s_{0}$ denotes the current sentence

\begin{tabular}{|c|c|c|c|}
\hline Full text & "Artificial intelligence" & "Civilization" & "Data compression" \\
\hline$s_{-3}$ & $\begin{array}{l}\text { The central problems (or goals) of AI } \\
\text { research include reasoning, } \\
\text { knowledge, planning, learning, } \\
\text { natural language processing, } \\
\text { perception and the ability to move } \\
\text { and manipulate objects. }\end{array}$ & $\begin{array}{l}\text { Historically, a civilization was a so-called } \\
\text { "advanced" culture in contrast to more } \\
\text { supposedly primitive cultures. }\end{array}$ & $\begin{array}{l}\text { Lossy compression reduces bits by } \\
\text { identifying unnecessary information } \\
\text { and removing it. }\end{array}$ \\
\hline$s_{-2}$ & $\begin{array}{l}\text { General intelligence is still among the } \\
\text { field's long-term goals. }\end{array}$ & $\begin{array}{l}\text { In this broad sense, a civilization contrasts with } \\
\text { non-centralized tribal societies, including the } \\
\text { cultures of nomadic pastoralists, egalitarian } \\
\text { horticultural subsistence neolithic societies or } \\
\text { hunter-gatherers. }\end{array}$ & $\begin{array}{l}\text { The process of reducing the size of a } \\
\text { data file is referred to as data } \\
\text { compression. }\end{array}$ \\
\hline$s_{-1}$ & $\begin{array}{l}\text { Currently popular approaches } \\
\text { include statistical methods, } \\
\text { computational intelligence and } \\
\text { traditional symbolic AI. }\end{array}$ & $\begin{array}{l}\text { As an uncountable noun, civilization also refers } \\
\text { to the process of a society developing into a } \\
\text { centralized, urbanized, stratified structure. }\end{array}$ & $\begin{array}{l}\text { In the context of data transmission, it } \\
\text { is called source coding in opposition } \\
\text { to channel coding. }\end{array}$ \\
\hline$s_{0}$ & $\begin{array}{l}\text { There are a large number of tools used } \\
\text { in AI, including versions of search and } \\
\text { mathematical optimization, logic, } \\
\text { methods based on probability and } \\
\text { economics, and many others. }\end{array}$ & $\begin{array}{l}\text { Civilizations are organized in densely populated } \\
\text { settlements divided into hierarchical social classes } \\
\text { with a ruling elite and subordinate urban and } \\
\text { rural populations, which engage in intensive } \\
\text { agriculture, mining, small-scale manufacture and } \\
\text { trade. }\end{array}$ & $\begin{array}{l}\text { Compression is useful because it helps } \\
\text { reduce resource usage, such as data } \\
\text { storage space or transmission capacity. }\end{array}$ \\
\hline$s_{1}$ & $\begin{array}{l}\text { The AI field draws upon computer } \\
\text { science, information engineering, } \\
\text { mathematics, psychology, linguistics, } \\
\text { philosophy, and many others. }\end{array}$ & $\begin{array}{l}\text { Civilization concentrates power, extending } \\
\text { human control over the rest of nature, including } \\
\text { over other human beings. }\end{array}$ & $\begin{array}{l}\text { Computational resources are } \\
\text { consumed in the compression process } \\
\text { and, usually, in the reversal of the } \\
\text { process. }\end{array}$ \\
\hline$s_{2}$ & $\begin{array}{l}\text { The field was founded on the claim } \\
\text { that human intelligence "can be so } \\
\text { precisely described that a machine } \\
\text { can be made to simulate it." }\end{array}$ & $\begin{array}{l}\text { Civilization, as its etymology (below) suggests, } \\
\text { is a concept originally linked to towns and } \\
\text { cities. }\end{array}$ & $\begin{array}{l}\text { Data compression is subject to a } \\
\text { space-time complexity trade-off. }\end{array}$ \\
\hline$s_{3}$ & $\begin{array}{l}\text { This raises philosophical arguments } \\
\text { about the nature of the mind and the } \\
\text { ethics of creating artificial beings } \\
\text { endowed with human-like } \\
\text { intelligence which are issues that } \\
\text { have been explored by myth, fiction } \\
\text { and philosophy since antiquity. }\end{array}$ & $\begin{array}{l}\text { The earliest emergence of civilizations is } \\
\text { generally associated with the final stages of the } \\
\text { Neolithic Revolution, culminating in the } \\
\text { relatively rapid process of urban revolution and } \\
\text { state formation, a political development } \\
\text { associated with the appearance of a governing } \\
\text { elite. }\end{array}$ & $\begin{array}{l}\text { For instance, a compression scheme } \\
\text { for video may require expensive } \\
\text { hardware for the video to be } \\
\text { decompressed fast enough to be } \\
\text { viewed as it is being decompressed, } \\
\text { and the option to decompress the } \\
\text { video in full before watching it may } \\
\text { be inconvenient or require additional } \\
\text { storage. }\end{array}$ \\
\hline
\end{tabular}

$s_{-1}$ to $s_{-3}$ denote the preceding sentences, and $s_{1}$ to $s_{3}$ denote the following sentences. "Full text" denotes the host document of these sentences.

for the current sentence $s_{0}$ shown in Table 5. For the RATM shown in Table 6, we do not use the topic distribution of the host document as $G_{0}$ since we just want to show the local relations among sentences and hence we manually set all the probabilities to sample from $G_{0}$ in step 2(c) of the RABP to be 0 . We also choose the topic distribution of the host document as $G_{0}$, and show the values of the attention signals of the global full text. The results are listed in the row "RATM-D" in Table 6. In "Artificial intelligence" and "Civilization," the attention signal values of the global texts are higher than the preceding sentences. The main reason is that the semantic meanings of the current sentences are more close to those of the document rather than the preceding sentences. We can see that current sentences in the two cases belong to the summarized descriptions, which share similar semantic distributions to the host documents. While, in "Data compression," one of the preceding sentences has high attention signal values as well as the global text. The last row of Table 6 shows the results of bi-RATM. Comparing with the attention signals in RATM-D, we find that some attention signal values of the subsequent sentences are higher than the preceding sentences in bi-RATM. Moreover, in "Artificial intelligence," the attention signal value of the first subsequent sentence is higher than the host document, which means that the topic distributions of 
Table 6. The Values of Attention Signals for Sentences in Some Documents of the Wikipedia Corpus

\begin{tabular}{|c|c|c|c|c|c|c|c|c|c|}
\hline & \multicolumn{3}{|c|}{ "Artificial intelligence" } & \multicolumn{3}{c|}{ "Civilization" } & \multicolumn{3}{c|}{ "Data compression" } \\
\cline { 2 - 10 } & RATM & RATM-D & bi-RATM & RATM & RATM-D & bi-RATM & RATM & RATM-D & bi-RATM \\
\hline Full text & - & 0.348576 & 0.196682 & - & 0.890223 & 0.501197 & - & 0.20342 & 0.300298 \\
$s_{-3}$ & 0.591437 & 0.469432 & 0.281189 & 0.187566 & 0.002313 & 0.004014 & 0.101403 & 0.077912 & 0.069342 \\
$s_{-2}$ & 0.219417 & 0.114332 & 0.010173 & 0.794651 & 0.106770 & 0.157762 & 0.869480 & 0.708424 & 0.289912 \\
$s_{-1}$ & 0.189146 & 0.06766 & 0.167660 & 0.017783 & 0.000694 & 0.000091 & 0.029107 & 0.010244 & 0.010244 \\
\hline$s_{1}$ & - & - & 0.231995 & - & - & 0.112204 & - & - & 0.00020 \\
$s_{2}$ & - & - & 0.010050 & - & - & 0.063739 & - & - & 0.021156 \\
$s_{3}$ & - & - & 0.102251 & - & - & 0.160993 & - & - & 0.308848 \\
\hline
\end{tabular}

The numbers are the values of attention signals of the contextual sentences and the host document for the current sentence $s_{0}$.

Table 7. The Ranked Topics Extracted by Different Models, Where Each Topic is Represented by Top 5 Words

\begin{tabular}{|c|c|c|}
\hline LDA & Manual Attentions & bi-RATM \\
\hline $\begin{array}{l}\text { <'data', 'processing', 'applications', 'information', } \\
\text { 'text'> } \\
\text { <'learning', 'machine', 'algorithm', 'algorithms', } \\
\text { 'neural'> } \\
\text { <'science', 'physics', 'field', 'scientific', 'sciences'> } \\
\text { <'American', 'radio', 'writer', 'television', 'show'> } \\
\text { <'television', 'show', 'aired', 'episode', 'episodes'> }\end{array}$ & $\begin{array}{c}\text { <'data', 'processing', 'applications', 'information', } \\
\text { 'text'> } \\
\text { <'learning', 'machine', 'algorithm', 'algorithms', } \\
\text { 'neural'> } \\
\text { <'science', 'physics', 'field', 'scientific', 'sciences'> } \\
\text { <'analysis',' 'model', 'methods', 'data', 'method'> } \\
\text { <'mathematics', 'mathematical', 'theory', } \\
\text { 'theorem', 'geometry'> }\end{array}$ & $\begin{array}{l}\text { <'learning', 'machine', 'algorithm', 'algorithms', } \\
\text { 'neural'> } \\
\text { <'data', 'processing', 'applications', 'information', } \\
\text { 'text'> } \\
\text { <'science', 'physics', 'field', 'scientific', 'sciences'> } \\
\text { <'analysis', 'model', 'methods', 'data', 'method'> } \\
\text { <'knowledge', 'information', 'management', } \\
\text { 'social', 'technology'> }\end{array}$ \\
\hline
\end{tabular}

the current sentence are similar to its subsequent sentence. The same phenomenon can be observed in the case of "Data compression." Thus, we find that the attention signal values are adjusted after we treat the document as bi-directional sequences of the sentences. The gain of the bi-RATM model is from the bi-directional sequences of the sentences and the global document. Our model can learn better attentions for the current sentence with the global or bi-directional local semantics, which not only verifies the assumption of the bi-RATM but also demonstrates its effectiveness.

9.4.3 Analysis on Extracted Topics. In this section, we analyze the topics learned by bi-RATM. Here we show the topics extracted by LDA and bi-RATM with the top five topics of sentences, and each topic is represented by the top 5 words ranked via their log-probabilities. We show the case of "Machine Learning" as shown in the introduction part, and the corresponding sentence is

"Machine learning is sometimes conflated with data mining, where the latter subfield focuses more on exploratory data analysis and is known as unsupervised learning."

We show the topics learned by LDA, which means the topic distribution of this sentence is obtained by its words but without considering the contextual sentences. Then, we show the topics learned by bi-RATM with two preceding and two following sentences as the contexts. The attentions learned by bi-RATM for the host document and the four neighbored sentences are (Full text: $\left.0.301, s_{-2}: 0.263, s_{-1}: 0.078, s_{1}: 0.162, s_{2}: 0.195\right)$. Last, we show the topics of this sentence by setting the attention signals manually in bi-RATM, for example, (Full text: 0.001, $s_{-2}: 0.470, s_{-1}: 0.442, s_{1}$ : $\left.0.084, s_{2}: 0.003\right)$.

Table 7 shows the results of extracted topics by those three methods. From the results, we can see that the topics extracted by LDA for the sentence are biased compared with those of extracted by bi-RATM. Compared with LDA and manual attentions, the topics extracted by bi-RATM with learned attentional signals are more fluent, coherent, and accurate. 


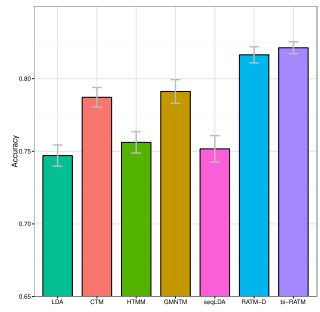

(a) $K=100$

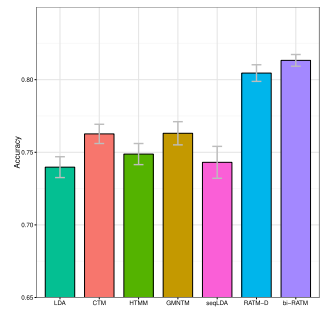

(b) $K=200$

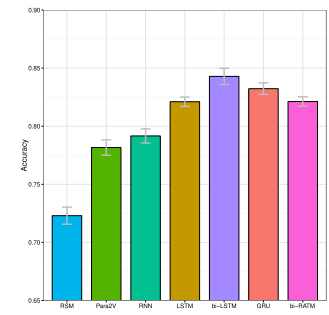

(c) $K=100$

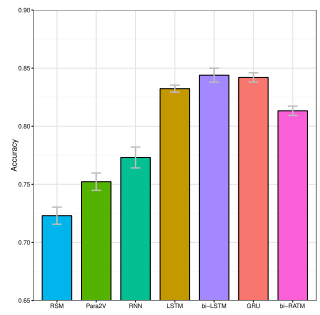

(d) $K=200$

Fig. 3. Classification results on the Wikipedia with five-fold cross-validation. (a) and (b) are the results of Bayesian topic models, and (c) and (d) are the results of neural networks.

\subsection{Experiments on Document Classification}

In this section, we evaluate the performance of different models on Wikipedia for the document classification task. We compare bi-RATM with the models which are based on Bayesian topic model, including LDA, CTM, HTMM, GMNTM, seqLDA, and RATM-D. For bi-RATM, we use $\beta$ generated by the LDA model to initialize the topic distributions over words. For the other models, we first extract the embeddings for all the documents in the corpus and then use the SVM with the RBF kernel as the classifier. The classification accuracy is used as the performance measure. We use $80 \%$ documents as the training samples and the rest $20 \%$ documents for testing. For all the models, we have two settings for the number of document features, namely 100 and 200, respectively. Moreover, neural-network-based models are included in the comparisons, including RSM, Para2V [36], RNN, LSTM, bi-LSTM, and GRU. Because the proposed model is an unsupervised model and thus for a fair comparison, we design an AutoEncoder framework for RNN, LSTM, bi-LSTM, and GRU. By taking RNN as an example, we design an RNN-RNN model where an RNN is built as the encoder and the other one as the decoder. The encoder takes the word embeddings as the input and the decoder is to rebuild the word embeddings, where the dimension of the word embedding is set to 100 . The word embeddings will be updated during the model training. The size of hidden layers is set to 100 and 200, respectively, and the dropout rate is set to 0.5 . We use the output of the encoder as the document features to train a classifier.

From the results shown in Figure 3, we can see that the performance of the bi-RATM model is significantly better than that of baseline methods based on the Bayesian topic model. One reason could be that the proposed bi-RATM uses not only the word counts in a document but also the bi-direction sequential information between sentences, leading to more effective embeddings for documents. Compared with baseline models based on neural networks, bi-RATM gets the competitive performance with the best baseline model, i.e., bi-LSTM, with $K$ as 100 and 200, respectively.

\subsection{Experiments on Online bi-RATM}

To compare the regular bi-RATM with the online version in terms of the convergence, we show the held-out perplexity as a function of the time on the large-scale Wikipedia corpus. The average batch size of the online algorithm is 2,000, and we run through all the 453 batches to obtain the model parameters. We set $k=0.75$ and $\tau_{0}=1024$. For a fair comparison, we implement these two algorithms in $C$ programming language and use the same settings $(K=200)$ and computing resources for both algorithms. Also, we compare with LDA and the online LDA, where the online LDA shares the same settings with the online bi-RATM. Besides, we also apply the online algorithm to RATM-D named online RATM-D, and compare with the regular RATM and the online RATM. 


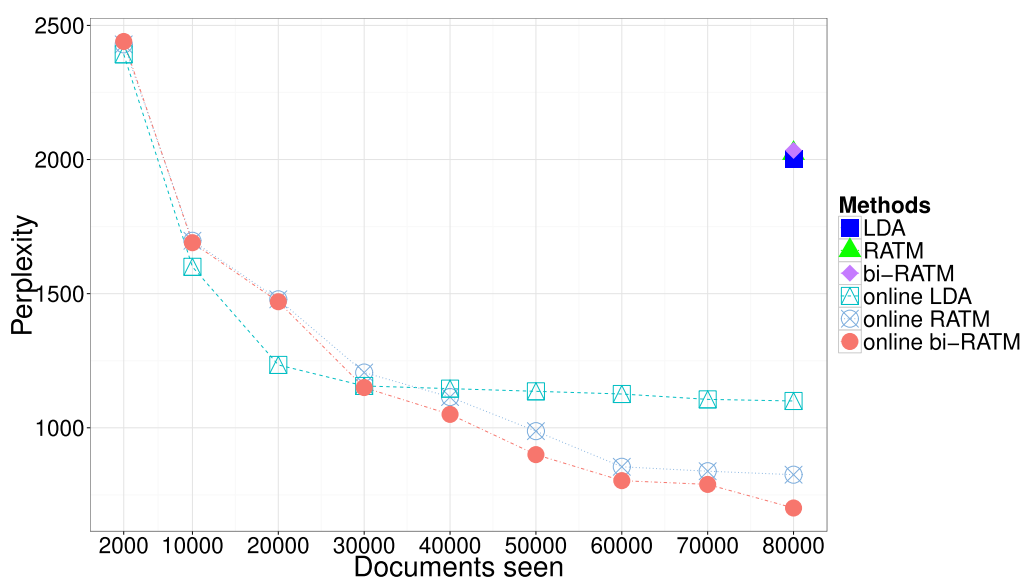

Fig. 4. The held-out perplexity on the Wikipedia corpus when documents constantly arrive.

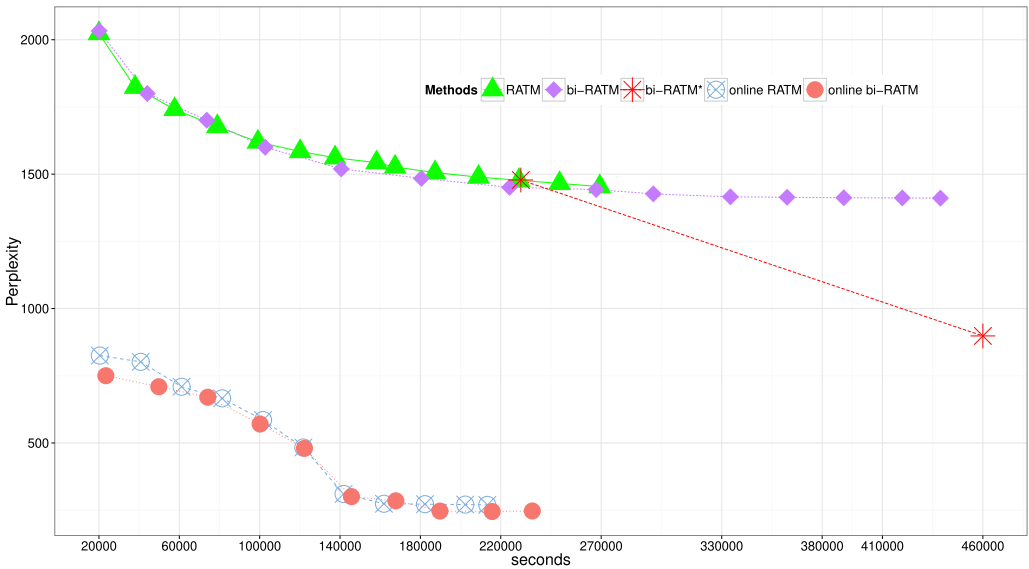

Fig. 5. The held-out perplexity on the Wikipedia corpus as a function of the CPU time.

Figure 4 shows the held-out perplexities of different algorithms when documents constantly arrive. According to Figure 4, RATM-D and bi-RATM do not converge since they have read all the 80,000 training documents once. In contrast, online bi-RATM and online RATM-D converges. Online LDA converges faster than online bi-RATM and online RATM-D, because online bi-RATM and online RATM-D have more parameters than online LDA to learn. However, online bi-RATM has better performance in terms of the perplexity with more and more documents coming than online LDA and online RATM-D. Note that the held-out perplexities of online RATM-D and online bi-RATM can become lower and lower with more documents coming even after 40 batches as shown in Figure 4.

Figure 5 summarizes the results on the held-out perplexity as a function of the CPU time after 20,000 seconds, which is just the time cost of one iteration of the regular bi-RATM on 40 batches of the documents. The regular RATM and bi-RATM still can handle on the 40 batches of the documents. We can see that the regular bi-RATM costs more time than the regular RATM in an iteration, 
while the bi-RATM converges faster. We also run bi-RATM with all the batches of the Wikipedia corpus as a whole dataset (denoted by bi-RATM ${ }^{*}$ in Figure 5), which cost about 230,000 seconds for one iteration. The online RATM and the online bi-RATM are shown every ten batches in Figure 5 . As shown in Figure 5, the online bi-RATM converges after 167,783 seconds, which is much faster than the regular bi-RATM and the bi-RATM*, even though their perplexities still decrease over time.

\section{CONCLUSION}

In this article, we propose a novel bi-RABP to handle sequential data. The proposed bi-RABP is a kind of Bayesian process that models the sequential data from two directions. Different from $\mathrm{RABP}$ considering the preceding sequences only, the bi-RABP takes advantage of the preceding and following sequential items together. Meanwhile, the attention signals are used to learn the latent relations among the local neighbored items, which significantly enhances the ability to capture the local information of the sequential data.

Thus, we apply it to document modeling by viewing a document as a bi-directional sequence of sentences, and develop a novel topic model, the bi-RATM, to handle sequences of sentences in a document. The proposed bi-RATM fully takes advantage of the bi-directional information to capture the latent topics of each sentence in a document. We evaluate the proposed model on topic modeling based on the following three measures: the held-out perplexity, topic coherence, and classification accuracy. Experiments demonstrate that the proposed model outperforms the state-of-the-art methods on document modeling. In addition, the visualization and analysis of the attention signals learned for sentences show that the proposed model has the capacity of capturing and extracting the latent relations among the neighbored sentences, which gives us a different perspective on document modeling. An online algorithm of the proposed bi-RATM is devised to handle large-scale document streams, and the experiments on large-scale Wikipedia suggest that our model is effective and easy to use.

This work opens up a fruitful perspective for document modeling on sentence-level under the framework of Bayesian topic modeling. The strength of the proposed model is to build the latent topic space through the sequences of sentences, which is not involved in the previous works. While, more information still need to be considered when modeling the sequences of sentences, such as the topic breaks, paragraphs, and syntactic information, which would be the future directions of our work to deal with complicated text data.

\section{A APPENDIX}

In this section, we derive the variational inference procedure and the parameter estimation procedure.

\section{A.1 Variational Lower Bound}

Recall that the fully-factorized variational distribution for sentence $\mathbf{s}_{j}$ with $N_{j}$ words in document $d$ is formulated as

$$
q\left(\vartheta^{d}, \epsilon^{j}, \mathbf{z} \mid \rho^{d} \xi, \gamma\right)=q\left(\vartheta^{d} \mid \rho^{d}\right) \prod_{j=1}^{S_{i}} q\left(\epsilon^{j} \mid \xi\right) \prod_{n=1}^{N_{j}} q\left(z_{n} \mid \gamma_{n}\right)
$$

where $\rho^{d}, \xi$, and $\gamma$ are variational parameters to be learned. 
Generally, based on the fully-factorized variational distribution and Equation (4), the lower bound of the log probability of the document $d$ can be computed based on the Jensen's inequality as

$$
\begin{aligned}
\mathcal{L}^{\mathrm{d}}\left(\beta, \pi, \alpha ; \xi, \gamma, \rho^{d}\right)= & \sum_{j=1}^{S_{i}} \mathcal{L}^{s_{j}}(\beta, \pi ; \xi, \gamma)+\mathbb{E}_{q}\left[\log p\left(\vartheta^{d} \mid \alpha\right)\right]-\mathbb{E}_{q}\left[\log q\left(\rho^{d}\right)\right] \\
= & \sum_{j=1}^{S_{i}} \mathbb{E}_{q}\left[\log p\left(\epsilon^{j} \mid \pi\right)\right]+\sum_{j=1}^{S_{i}} \sum_{n=1}^{N_{j}} \mathbb{E}_{q}\left[\log p\left(z_{n} \mid \epsilon^{j}, \theta^{2 C+1}\right)\right]+\sum_{j=1}^{S_{i}} \sum_{n=1}^{N_{j}} \mathbb{E}_{q}\left[\log p\left(w_{n} \mid z_{n}, \beta\right)\right] \\
& -\sum_{j=1}^{S_{i}} \mathbb{E}_{q}\left[\log q\left(\epsilon^{j}\right)\right]-S_{i} \cdot \mathbb{E}_{q}[\log q(\mathbf{z})]+\mathbb{E}_{q}\left[\log p\left(\vartheta^{d} \mid \alpha\right)\right]-\mathbb{E}_{q}\left[\log q\left(\rho^{d}\right)\right]
\end{aligned}
$$

Note that, even though it is difficult to compute $\mathbb{E}_{q}\left[\log p\left(z_{n} \mid \epsilon^{j}, \theta^{2 C+1}\right)\right]$, we can obtain its lowerbound for sentence $s_{j}$ as

$$
\begin{aligned}
\sum_{n=1}^{N_{j}} \mathbb{E}_{q}\left[\log p\left(z_{n} \mid \epsilon^{j}, \theta^{2 C+1}\right)\right] & =\sum_{n=1}^{N_{j}} \sum_{k=1}^{K} \gamma_{n k} \mathbb{E}_{q}\left[\log \left(\epsilon^{j} \times \theta^{2 C+1}\right)_{k}\right] \\
& \geq \sum_{n=1}^{N_{j}} \sum_{k=1}^{K} \gamma_{n k} \sum_{l=1}^{2 C+1} \log \theta_{l k}^{2 C+1} \mathbb{E}_{q}\left[\epsilon_{l}^{j}\right] \\
& =\sum_{n=1}^{N_{j}} \sum_{k=1}^{K} \gamma_{n k} \sum_{l=1}^{2 C+1} \log \theta_{l k}^{2 C+1} \frac{\xi_{l}}{\sum_{l^{\prime}} \xi_{l^{\prime}}},
\end{aligned}
$$

where $\theta_{l k}^{2 C+1}$ denotes the $k$-th element in the $l$-th row of $\theta^{2 C+1}$ and the last equality holds since the expectation of a Dirichlet random variable can be computed as $\mathbb{E}_{q}\left[\epsilon_{l}^{j}\right]=\frac{\xi_{l}}{\sum_{l^{\prime}} \xi_{l^{\prime}}}$. In Equation (18), the computation of $\epsilon^{j} \times \theta^{2 C+1}$ gives a $K$-dimensional vector, which is the topic distribution of the current sentence $j$. Note that the rows in $\theta^{2 C+1}$ are fixed which can be treated as constants when we simplify $\mathbb{E}_{q}\left[\log \left(\epsilon^{j} \times \theta^{2 C+1}\right)_{k}\right]$ for sentence $j$ via the Jensen's inequality. For the document level, we need to decompose $\theta^{2 C+1}$ for the variable $\vartheta^{d}$.

Then we can formulate the $\mathcal{L}^{s_{j}}(\beta, \pi ; \xi, \gamma)$ for a sentence in terms of the model parameters $\pi, \beta$ and the variational parameters $\xi, \gamma$ as

$$
\begin{aligned}
\mathcal{L}^{s_{j}}(\beta, \pi ; \xi, \gamma)= & \sum_{n=1}^{N_{j}} \sum_{k=1}^{K} \gamma_{n k} \sum_{l=1}^{2 C+1} \log \theta_{l k}^{2 C+1} \frac{\xi_{l}}{\sum_{l^{\prime}=1}^{2 C+1} \xi_{l^{\prime}}}+\log \Gamma\left(\sum_{l=1}^{2 C+1} \pi_{l}\right)+\sum_{l=1}^{2 C+1}\left(\pi_{l}-1\right)\left(\Psi\left(\xi_{l}\right)-\Psi\left(\sum_{l^{\prime}=1}^{2 C+1} \xi_{l^{\prime}}\right)\right) \\
& -\sum_{l^{\prime}=1}^{2 C+1} \log \Gamma\left(\pi_{l^{\prime}}\right)+\sum_{n=1}^{N_{j}} \sum_{k=1}^{K} \sum_{v=1}^{V} \gamma_{n k} w_{n}^{v} \log \beta_{k v}-\sum_{n=1}^{N_{j}} \sum_{k=1}^{K} \gamma_{n k} \log \gamma_{n k}-\log \Gamma\left(\sum_{l=1}^{2 C+1} \xi_{l}\right)+\sum_{l=1}^{2 C+1} \log \Gamma\left(\xi_{l}\right) \\
& -\sum_{l=1}^{2 C+1}\left(\xi_{l}-1\right)\left(\Psi\left(\xi_{l}\right)-\Psi\left(\sum_{l^{\prime}=1}^{2 C+1} \xi_{l^{\prime}}\right)\right),
\end{aligned}
$$

where $V$ is the size of the dictionary. 
Finally, by plugging Equation (18) into Equation (17), $\mathcal{L}^{\mathrm{d}}\left(\beta, \pi, \alpha ; \xi, \gamma, \rho^{d}\right)$ can be computed as

$$
\begin{aligned}
& \mathcal{L}^{\mathrm{d}}\left(\beta, \pi, \alpha ; \xi, \gamma, \rho^{d}\right) \\
= & S_{i} \cdot \log \Gamma\left(\sum_{l=1}^{2 C+1} \pi_{l}\right)-S_{i} \cdot \sum_{l^{\prime}=1}^{2 C+1} \log \Gamma\left(\pi_{l^{\prime}}\right)+S_{i} \cdot \sum_{l=1}^{2 C+1}\left(\pi_{l}-1\right)\left(\Psi\left(\xi_{l}\right)-\Psi\left(\sum_{l^{\prime}=1}^{2 C+1} \xi_{l^{\prime}}\right)\right) \\
& +\sum_{j=1}^{S_{i}} \sum_{n=1}^{N_{j}} \sum_{k=1}^{K} \gamma_{n k} \sum_{l=1}^{2 C+1} \log \theta_{l k}^{2 C+1} \frac{\xi_{l}}{\sum_{l^{\prime}=1}^{2 C+1} \xi_{l^{\prime}}}+\sum_{j=1}^{S_{i}} \sum_{n=1}^{N_{j}} \sum_{k=1}^{K} \sum_{v=1}^{V} \gamma_{n k} w_{n}^{v} \log \beta_{k v} \\
& -S_{i} \cdot \log \Gamma\left(\sum_{l=1}^{2 C+1} \xi_{l}\right)+S_{i} \cdot \sum_{l=1}^{2 C+1} \log \Gamma\left(\xi_{l}\right)-S_{i} \cdot \sum_{l=1}^{2 C+1}\left(\xi_{l}-1\right)\left(\Psi\left(\xi_{l}\right)-\Psi\left(\sum_{l^{\prime}=1}^{2 C+1} \xi_{l^{\prime}}\right)\right)-\sum_{j=1}^{S_{i}} \sum_{n=1}^{N_{j}} \sum_{k=1}^{K} \gamma_{n k} \log \gamma_{n k} \\
& +\log \Gamma\left(\sum_{k=1}^{K} \alpha_{k}\right)-\sum_{k=1}^{K} \log \Gamma\left(\alpha_{k}\right)+\sum_{k=1}^{K}\left(\alpha_{k}-1\right)\left(\Psi\left(\rho^{\mathrm{d}^{i}}\right)-\Psi\left(\sum_{k=1}^{K} \rho^{\mathrm{d}^{i}}\right)\right) \\
& -\log \Gamma\left(\sum_{k=1}^{K} \rho_{k}^{\mathrm{d}^{i}}\right)+\sum_{k=1}^{K} \log \Gamma\left(\rho_{k}^{\mathrm{d}^{i}}\right)-\sum_{k=1}^{K}\left(\rho_{k}^{\mathrm{d}^{i}}-1\right)\left(\Psi\left(\rho^{\mathrm{d}^{i}}\right)-\Psi\left(\sum_{k=1}^{K} \rho^{\mathrm{d}^{i}}\right)\right) .
\end{aligned}
$$

Variational parameters in this lower bound include $\xi, \gamma$, and $\rho^{d}$. In the following, we show how to maximize this lower bound w.r.t these variational parameters.

A.1.1 $\xi$. Note that, the attention signal $\xi$ is related to the corresponding sentence. Thus, we maximize Equation (19) w.r.t $\xi$ for each sentence. For sentence $s_{j}$, the corresponding objective function based on Equation (19) is formulated as

$$
\begin{aligned}
\mathcal{L}_{[\xi]}^{s_{j}}= & \sum_{l=1}^{2 C+1}\left(\pi_{l}-1\right)\left(\Psi\left(\xi_{l}\right)-\Psi\left(\sum_{l^{\prime}=1}^{2 C+1} \xi_{l^{\prime}}\right)\right)-\log \Gamma\left(\sum_{l=1}^{2 C+1} \xi_{l}\right)+\sum_{l=1}^{2 C+1} \log \Gamma\left(\xi_{l}\right)-\sum_{l=1}^{2 C+1}\left(\xi_{l}-1\right)\left(\Psi\left(\xi_{l}\right)\right. \\
& \left.-\Psi\left(\sum_{l^{\prime}=1}^{2 C+1} \xi_{l^{\prime}}\right)\right)+\sum_{n=1}^{N_{j}} \sum_{k=1}^{K} \gamma_{n k} \sum_{l=1}^{2 C+1} \log \theta_{l k}^{2 C+1} \frac{\xi_{l}}{\sum_{l^{\prime}=1}^{2 C+1} \xi_{l^{\prime}}}
\end{aligned}
$$

where we ignore $S_{i}$. Then $\mathcal{L}_{[\xi]}^{s_{j}}$ can be simplified as

$$
\begin{aligned}
\mathcal{L}_{[\xi]}^{s_{j}}= & \sum_{l=1}^{2 C+1}\left(\pi_{l}-\xi_{l}\right)\left(\Psi\left(\xi_{l}\right)-\Psi\left(\sum_{l^{\prime}=1}^{2 C+1} \xi_{l^{\prime}}\right)\right)-\log \Gamma\left(\sum_{l=1}^{2 C+1} \xi_{l}\right)+\sum_{l=1}^{2 C+1} \log \Gamma\left(\xi_{l}\right) \\
& +\sum_{n=1}^{N_{j}} \sum_{k=1}^{K} \gamma_{n k} \sum_{l=1}^{2 C+1} \log \theta_{l k}^{2 C+1} \frac{\xi_{l}}{\sum_{l^{\prime}=1}^{2 C+1} \xi_{l^{\prime}}} .
\end{aligned}
$$

We compute the derivative w.r.t $\xi$ as

$$
\begin{aligned}
\mathcal{L}^{\prime}\left(\xi_{l}\right)= & \Psi^{\prime}\left(\xi_{l}\right)\left(\sum_{i=1}^{2 C+1} \pi_{i}-\xi_{l}\right)+\sum_{n=1}^{N_{j}} \sum_{k=1}^{K} \gamma_{n k}\left(\frac{\log \theta_{l k}^{2 C+1}\left(\sum_{j=1}^{2 C+1} \xi_{j}\right)-\sum_{i=1}^{2 C+1} \log \theta_{i k}^{2 C+1} \xi_{i}}{\sum_{j^{\prime}=1}^{2 C+1} \xi_{j^{\prime}}{ }^{2}}\right) \\
& -\Psi^{\prime}\left(\sum_{i=1}^{2 C+1} \xi_{i}\right) \sum_{l=1}^{2 C+1}\left(\sum_{i=1}^{2 C+1} \pi_{i}-\xi_{l}\right) .
\end{aligned}
$$


A.1.2 $\gamma$. We aim to maximize Equation (19) w.r.t $\gamma$. The objective function for $\gamma$ is formulated as

$$
\mathcal{L}_{[\gamma]}^{s_{j}}=\sum_{n=1}^{N_{j}} \sum_{k=1}^{K} \gamma_{n k} \sum_{l=1}^{2 C+1} \log \theta_{l k}^{2 C+1} \frac{\xi_{l}}{\sum_{l^{\prime}=1}^{2 C+1} \xi_{l^{\prime}}}-\sum_{n=1}^{N_{j}} \sum_{k=1}^{K} \gamma_{n k} \log \gamma_{n k}+\sum_{n=1}^{N_{j}} \sum_{k=1}^{K} \sum_{v=1}^{V} \gamma_{n k} w_{n}^{v} \log \beta_{k v} .
$$

Similarly, we maximize Equation (19) w.r.t $\gamma$ in the sentence level. To maximize w.r.t $\gamma$, we define the Lagrangian as

$$
\begin{aligned}
\mathcal{L}_{[\gamma]}^{s_{j}}= & \sum_{n=1}^{N_{j}} \sum_{k=1}^{K} \gamma_{n k} \sum_{l=1}^{2 C+1} \log \theta_{l k}^{2 C+1} \frac{\xi_{l}}{\sum_{l^{\prime}=1}^{2 C+1} \xi_{l^{\prime}}}-\sum_{n=1}^{N_{j}} \sum_{k=1}^{K} \gamma_{n k} \log \gamma_{n k}+\sum_{n=1}^{N_{j}} \sum_{k=1}^{K} \sum_{v=1}^{V} \gamma_{n k} w_{n}^{v} \log \beta_{k v} \\
& +\sum_{n=1}^{N_{j}} \lambda_{n}\left(\sum_{k=1}^{K} \gamma_{n k}-1\right) .
\end{aligned}
$$

By setting the derivative w.r.t $\gamma$ to zero, we obtain the update equation as

$$
\gamma_{n k} \propto \beta_{k, v^{w_{n}}} \exp \sum_{l=1}^{2 C+1} \log \theta_{l k}^{2 C+1} \frac{\xi_{l}}{\sum_{l^{\prime}} \xi_{l^{\prime}}} .
$$

A.1.3 $\rho^{\mathrm{d}^{i}}$. For document $d^{i}$, we need to reformulate Equation (18) as

$$
\begin{aligned}
& \sum_{n=1}^{N_{j}} \mathbb{E}_{q}\left[\log p\left(z_{n} \mid \epsilon^{j}, \theta^{2 C+1}\right)\right] \\
= & \sum_{n=1}^{N_{j}} \sum_{k=1}^{K} \gamma_{n k} \mathbb{E}_{q}\left[\log \left(\sum_{l}^{2 C} \epsilon_{l} \theta_{k}^{2 C}+\epsilon_{C} \cdot \vartheta_{k}\right)\right] \\
\geq & \sum_{n=1}^{N_{j}} \sum_{k=1}^{K} \gamma_{n k}\left(\sum_{l=1}^{2 C} \log \theta_{l k}^{2 C} \mathbb{E}_{q}\left[\epsilon_{l}^{j}\right]+\mathbb{E}_{q}\left[\epsilon_{C} \cdot \log \vartheta_{k}\right]\right) \\
= & \sum_{n=1}^{N_{j}} \sum_{k=1}^{K} \gamma_{n k} \sum_{l=1}^{2 C} \log \theta_{l k}^{2 C} \frac{\xi_{l}}{\sum_{l^{\prime}} \xi_{l^{\prime}}}+\sum_{n=1}^{N_{j}} \sum_{k=1}^{K} \gamma_{n k} \frac{\xi_{C}}{\sum_{l}^{C} \xi_{l}} \cdot\left(\Psi\left(\rho^{\mathrm{d}^{i}}\right)-\Psi\left(\sum_{k=1}^{K} \rho^{\mathrm{d}^{i}}\right)\right),
\end{aligned}
$$

where

$$
\mathbb{E}_{q}\left[\epsilon_{C} \cdot \log \vartheta_{k}\right]=\mathbb{E}_{q}\left[\epsilon_{C}\right] \cdot \mathbb{E}_{q}\left[\log \vartheta_{k}\right]=\frac{\xi_{C}}{\sum_{l}^{C} \xi_{l}} \cdot\left(\Psi\left(\rho^{\mathrm{d}^{i}}\right)-\Psi\left(\sum_{k=1}^{K} \rho^{\mathrm{d}^{i}}\right)\right)
$$

Thus, based on Equations (19) and (24), the objective function for $\rho^{d}$ is formulated as

$$
\begin{aligned}
\mathcal{L}_{\left[\rho^{d}\right]}= & \sum_{k=1}^{K}\left(\alpha_{k}-1\right)\left(\Psi\left(\rho^{\mathrm{d}^{i}}\right)-\Psi\left(\sum_{k=1}^{K} \rho^{\mathrm{d}^{i}}\right)\right)-\log \Gamma\left(\sum_{k=1}^{K} \rho_{k}^{\mathrm{d}^{i}}\right)+\sum_{k=1}^{K} \log \Gamma\left(\rho_{k}^{\mathrm{d}^{i}}\right)-\sum_{k=1}^{K}\left(\rho_{k}^{\mathrm{d}^{i}}-1\right)\left(\Psi\left(\rho^{\mathrm{d}^{i}}\right)-\Psi\left(\sum_{k=1}^{K} \rho^{\mathrm{d}^{i}}\right)\right) \\
& +\sum_{j=1}^{S_{i}} \sum_{n=1}^{N_{j}} \sum_{k=1}^{K} \gamma_{n k} \frac{\xi_{C}}{\sum_{l}^{C} \xi_{l}} \cdot\left(\Psi\left(\rho^{\mathrm{d}^{i}}\right)-\Psi\left(\sum_{k=1}^{K} \rho^{\mathrm{d}^{i}}\right)\right),
\end{aligned}
$$

which can be simplified as

$$
\mathcal{L}_{\left[\rho^{d}\right]}=\sum_{k=1}^{K}\left(\alpha_{k}+\sum_{j=1}^{S_{i}} \sum_{n=1}^{N_{j}} \gamma_{n k} \frac{\xi_{C}}{\sum_{l}^{C} \xi_{l}}-\rho_{k}^{\mathrm{d}^{i}}\right)\left(\Psi\left(\rho^{\mathrm{d}^{i}}\right)-\Psi\left(\sum_{k=1}^{K} \rho^{\mathrm{d}^{i}}\right)\right)-\log \Gamma\left(\sum_{k=1}^{K} \rho_{k}^{\mathrm{d}^{i}}\right)+\sum_{k=1}^{K} \log \Gamma\left(\rho_{k}^{\mathrm{d}^{i}}\right) .
$$


We compute the derivative w.r.t $\rho^{\mathrm{d}^{i}}$ as

$$
\mathcal{L}_{\left[\rho^{\left.\mathrm{d}^{i}\right]}\right.}^{\prime}=\Psi^{\prime}\left(\rho^{\mathrm{d}^{i}}\right)\left(\alpha_{k}+\sum_{j=1}^{S_{i}} \sum_{n=1}^{N_{j}} \gamma_{n k} \frac{\xi_{C}}{\sum_{l}^{C} \xi_{l}}-\rho_{k}^{\mathrm{d}^{i}}\right)-\Psi^{\prime}\left(\sum_{k=1}^{K} \rho^{\mathrm{d}^{i}}\right) \sum_{k=1}^{K}\left(\alpha_{k}+\sum_{j=1}^{S_{i}} \sum_{n=1}^{N_{j}} \gamma_{n k} \frac{\xi_{C}}{\sum_{l}^{C} \xi_{l}}-\rho_{k}^{\mathrm{d}^{i}}\right) .
$$

Setting this equation to zero yields a solution as

$$
\rho_{k}^{\mathrm{d}^{i}}=\alpha_{k}+\sum_{j=1}^{S_{i}} \sum_{n=1}^{N_{j}} \gamma_{n k} \cdot \frac{\xi_{C}}{\sum_{l}^{C} \xi_{l}}
$$

\section{A.2 Model Parameter}

The model parameters include $\beta$ and $\pi$. Here we show the details about the learning of $\beta$ and $\pi$.

A.2.1 $\beta$. To maximize w.r.t $\beta$, we define the Lagrangian as

$$
\mathcal{L}_{[\beta]}^{d}=\sum_{i=1}^{M} \sum_{j=1}^{S_{i}} \sum_{n=1}^{N_{j}} \sum_{k=1}^{K} \sum_{v=1}^{V} \gamma_{n k} w_{n}^{v} \log \beta_{k v}+\sum_{k=1}^{K} \lambda_{k}\left(\sum_{v=1}^{V} \beta_{k v}-1\right) .
$$

By setting the derivative w.r.t $\beta$ to zero, we find the following solution as

$$
\beta_{k v}=\sum_{i=1}^{M} \sum_{j=1}^{S_{i}} \sum_{n=1}^{N_{j}} \gamma_{n k} \cdot w_{n}^{v}
$$

A.2.2 $\pi$. The objective function for $\pi$ is formulated as

$$
\mathcal{L}_{[\pi]}^{d}=\sum_{i=1}^{M} \sum_{j=1}^{S_{i}}\left(\log \Gamma\left(\sum_{l=1}^{2 C+1} \pi_{l}\right)-\sum_{l^{\prime}=1}^{2 C+1} \log \Gamma\left(\pi_{l^{\prime}}\right)+\sum_{l=1}^{2 C+1}\left(\pi_{l}-1\right)\left(\Psi\left(\xi_{l}\right)-\Psi\left(\sum_{l^{\prime}=1}^{2 C+1} \xi_{l^{\prime}}\right)\right)\right) .
$$

We can compute the derivative w.r.t $\pi_{l}$ as

$$
\mathcal{L}_{\left[\pi_{l}\right]}^{\prime}=\sum_{i=1}^{M} S_{i}\left(\Psi\left(\sum_{l=1}^{2 C+1} \pi_{l}\right)-\Psi\left(\pi_{l}\right)\right)+\sum_{i=1}^{M} S_{i}\left(\Psi\left(\xi_{l}\right)-\Psi\left(\sum_{l^{\prime}=1}^{2 C+1} \xi_{l^{\prime}}\right)\right) .
$$

\section{ACKNOWLEDGMENTS}

We also thank the great support of Prof. Qiang Yang from Department of Computer Science and Engineering, Hong Kong University of Science and Technology.

\section{REFERENCES}

[1] Amr Ahmed and Eric P. Xing. 2008. Dynamic non-parametric mixture models and the recurrent Chinese restaurant process: With applications to evolutionary clustering. In Proceedings of the 2008 SIAM International Conference on Data Mining. 219-230.

[2] Mohammadreza Babaee, Duc Tung Dinh, and Gerhard Rigoll. 2018. A deep convolutional neural network for video sequence background subtraction. Pattern Recognition 76 (April 2018), 635-649.

[3] Dzmitry Bahdanau, Kyunghyun Cho, and Yoshua Bengio. 2015. Neural machine translation by jointly learning to align and translate. In 3rd International Conference on Learning Representations, San Diego, CA, USA, May 7-9, 2015, Conference Track Proceedings.

[4] David M. Blei. 2012. Probabilistic topic models. Communications of the ACM 55, 4 (2012), 77-84. DOI : https://doi.org/ $10.1145 / 2133806.2133826$

[5] David M. Blei, Thomas L. Griffiths, and Michael I. Jordan. 2010. The nested Chinese restaurant process and Bayesian nonparametric inference of topic hierarchies. Journal of the ACM 57, 2 (2010), 30. DOI : https://doi.org/10.1145/1667053. 1667056 
[6] David M. Blei and John D. Lafferty. 2006. Correlated topic models. In Proceedings of the Advances in Neural Information Processing Systems. 147-154.

[7] David M. Blei, Andrew Y. Ng, and Michael I. Jordan. 2003. Latent Dirichlet allocation. fournal of Machine Learning Research 3, Jan (2003), 993-1022.

[8] Léon Bottou and Olivier Bousquet. 2008. The tradeoffs of large scale learning. In Proceedings of the Advances in Neural Information Processing Systems. 161-168.

[9] Jordan L. Boyd-Graber and David M. Blei. 2009. Syntactic topic models. In Proceedings of the Advances in Neural Information Processing Systems. 185-192.

[10] Peter F. Brown, Peter V. Desouza, Robert L. Mercer, Vincent J. Della Pietra, and Jenifer C. Lai. 1992. Class-based $n$-gram models of natural language. Computational Linguistics 18, 4 (1992), 467-479.

[11] Deng Cai, Qiaozhu Mei, Jiawei Han, and Chengxiang Zhai. 2008. Modeling hidden topics on document manifold. In Proceedings of the 17th ACM Conference on Information and Knowledge Management. 911-920.

[12] Olivier Cappé and Eric Moulines. 2009. On-line expectation-maximization algorithm for latent data models. fournal of the Royal Statistical Society: Series B (Statistical Methodology) 71, 3 (2009), 593-613.

[13] Jianguo Chen, Kenli Li, Kashif Bilal, Keqin Li, S. Yu Philip, et al. 2018. A bi-layered parallel training architecture for large-scale convolutional neural networks. IEEE Transactions on Parallel and Distributed Systems 30, 5 (2018), 965-976.

[14] Jianguo Chen, Kenli Li, Zhuo Tang, Kashif Bilal, Shui Yu, Chuliang Weng, and Keqin Li. 2016. A parallel random forest algorithm for big data in a spark cloud computing environment. IEEE Transactions on Parallel and Distributed Systems 28, 4 (2016), 919-933.

[15] Yong Chen, Junjie Wu, Jianying Lin, Rui Liu, Hui Zhang, and Zhiwen Ye. 2019. Affinity regularized non-negative matrix factorization for lifelong topic modeling. IEEE Transactions on Knowledge and Data Engineering 32, 7 (2019), 1249-1262. DOI : https://doi.org/10.1109/TKDE.2019.2904687

[16] Zhiyuan Chen and Bing Liu. 2014. Topic modeling using topics from many domains, lifelong learning and big data. In Proceedings of the International Conference on Machine Learning. 703-711.

[17] Jan K. Chorowski, Dzmitry Bahdanau, Dmitriy Serdyuk, Kyunghyun Cho, and Yoshua Bengio. 2015. Attention-based models for speech recognition. In Proceedings of the Advances in Neural Information Processing Systems. 577-585.

[18] Rajarshi Das, Manzil Zaheer, and Chris Dyer. 2015. Gaussian LDA for topic models with word embeddings. In Proceedings of the 53rd Annual Meeting of the Association for Computational Linguistics and the 7th International foint Conference on Natural Language Processing. 795-804.

[19] Adji B. Dieng, Chong Wang, Jianfeng Gao, and John Paisley. 2017. TopicRnn: A recurrent neural network with longrange semantic dependency. Proceedings of the 5th International Conference on Learning Representations.

[20] Chris Ding, Tao Li, and Wei Peng. 2008. On the equivalence between non-negative matrix factorization and probabilistic latent semantic indexing. Computational Statistics and Data Analysis 52, 8 (2008), 3913-3927.

[21] Lan Du, Wray Buntine, Huidong Jin, and Changyou Chen. 2012. Sequential latent Dirichlet allocation. Knowledge and Information Systems 31, 3 (2012), 475-503.

[22] Nan Du, Mehrdad Farajtabar, Amr Ahmed, Alexander J. Smola, and Le Song. 2015. Dirichlet-Hawkes processes with applications to clustering continuous-time document streams. In Proceedings of the 21th ACM SIGKDD International Conference on Knowledge Discovery and Data Mining. 219-228.

[23] Li Fei-Fei and Pietro Perona. 2005. A Bayesian hierarchical model for learning natural scene categories. In Proceedings of the 2005 IEEE Computer Society Conference on Computer Vision and Pattern Recognition. Vol. 2. 524-531.

[24] Volkmar Frinken, Andreas Fischer, R. Manmatha, and Horst Bunke. 2011. A novel word spotting method based on recurrent neural networks. IEEE Transactions on Pattern Analysis and Machine Intelligence 34, 2 (2011), 211-224.

[25] Zhe Gan, Changyou Chen, Ricardo Henao, David Carlson, and Lawrence Carin. 2015. Scalable deep Poisson factor analysis for topic modeling. In Proceedings of the International Conference on Machine Learning. 1823-1832.

[26] Thomas L. Griffiths, Mark Steyvers, David M. Blei, and Joshua B. Tenenbaum. 2005. Integrating topics and syntax. In Proceedings of the Advances in Neural Information Processing Systems. 537-544.

[27] Amit Gruber, Yair Weiss, and Michal Rosen-Zvi. 2007. Hidden topic Markov models. In Proceedings of the 11th International Conference on Artificial Intelligence and Statistics. 163-170.

[28] Ricardo Henao, Zhe Gan, James Lu, and Lawrence Carin. 2015. Deep poisson factor modeling. In Proceedings of the Advances in Neural Information Processing Systems. 2800-2808.

[29] Geoffrey E. Hinton and Ruslan R. Salakhutdinov. 2009. Replicated softmax: An undirected topic model. In Proceedings of the Advances in Neural Information Processing Systems. 1607-1614.

[30] Matthew D. Hoffman, David M. Blei, and Francis R. Bach. 2010. Online learning for latent dirichlet allocation. In Proceedings of the Advances in Neural Information Processing Systems. 856-864.

[31] Thomas Hofmann. 1999. Probabilistic latent semantic indexing. In Proceedings of the 22nd Annual International ACM SIGIR Conference on Research and Development in Information Retrieval. 50-57. 
[32] Michael I. Jordan, Zoubin Ghahramani, Tommi Jaakkola, and Lawrence K. Saul. 1999. An introduction to variational methods for graphical models. Machine Learning 37, 2 (1999), 183-233.

[33] Ryan Kiros, Yukun Zhu, Ruslan R. Salakhutdinov, Richard Zemel, Raquel Urtasun, Antonio Torralba, and Sanja Fidler. 2015. Skip-thought vectors. In Proceedings of the Advances in Neural Information Processing Systems. 3294-3302.

[34] Siwei Lai, Liheng Xu, Kang Liu, and Jun Zhao. 2015. Recurrent convolutional neural networks for text classification. In Proceedings of the 29th AAAI Conference on Artificial Intelligence. 2267-2273.

[35] Hugo Larochelle and Stanislas Lauly. 2012. A neural autoregressive topic model. In Proceedings of the Advances in Neural Information Processing Systems. 2708-2716.

[36] Quoc Le and Tomas Mikolov. 2014. Distributed representations of sentences and documents. In Proceedings of the International Conference on Machine Learning. 1188-1196.

[37] Chenliang Li, Haoran Wang, Zhiqian Zhang, Aixin Sun, and Zongyang Ma. 2016. Topic modeling for short texts with auxiliary word embeddings. In Proceedings of the 39th International ACM SIGIR conference on Research and Development in Information Retrieval. 165-174.

[38] Shuangyin Li, Yu Zhang, Rong Pan, Mingzhi Mao, and Yang Yang. 2017. Recurrent attentional topic model. In Proceedings of the 31st AAAI Conference on Artificial Intelligence. Vol. 17. 3223-3229.

[39] Percy Liang and Dan Klein. 2009. Online EM for unsupervised models. In Proceedings of the 2009 Annual Conference of the North American Chapter of the Association for Computational Linguistics: Human Language Technologies. 611-619.

[40] Rui Lin, Shujie Liu, Muyun Yang, Mu Li, Ming Zhou, and Sheng Li. 2015. Hierarchical recurrent neural network for document modeling. In Proceedings of the 2015 Conference on Empirical Methods in Natural Language Processing. 899-907.

[41] Wang Ling, Lin Chu-Cheng, Yulia Tsvetkov, and Silvio Amir. 2015. Not all contexts are created equal: Better word representations with variable attention. In Proceedings of the 2015 Conference on Empirical Methods in Natural Language Processing. 1367-1372.

[42] Benjamin M. Marlin. 2004. Modeling user rating profiles for collaborative filtering. In Proceedings of the Advances in Neural Information Processing Systems. 627-634.

[43] Jon D. Mcauliffe and David M. Blei. 2008. Supervised topic models. In Proceedings of the Advances in Neural Information Processing Systems. 121-128.

[44] Tomas Mikolov, Martin Karafiát, Lukas Burget, Jan Cernockỳ, and Sanjeev Khudanpur. 2010. Recurrent neural network based language model. In Proceedings of the 11th Annual Conference of the International Speech Communication Association.

[45] Tomas Mikolov, Ilya Sutskever, Kai Chen, Greg S. Corrado, and Jeff Dean. 2013. Distributed representations of words and phrases and their compositionality. In Proceedings of the Advances in Neural Information Processing Systems. 3111-3119.

[46] Tomas Mikolov, Wen-tau Yih, and Geoffrey Zweig. 2013. Linguistic regularities in continuous space word representations. In Proceedings of the 2013 Conference of the North American Chapter of the Association for Computational Linguistics: Human Language Technologies. 746-751.

[47] David Mimno, Hanna M. Wallach, Edmund Talley, Miriam Leenders, and Andrew McCallum. 2011. Optimizing semantic coherence in topic models. In Proceedings of the Conference on Empirical Methods in Natural Language Processing. Association for Computational Linguistics, 262-272.

[48] David Newman, Arthur Asuncion, Padhraic Smyth, and Max Welling. 2009. Distributed algorithms for topic models. Journal of Machine Learning Research 10, 8 (2009), 1801-1828.

[49] David Newman, Youn Noh, Edmund Talley, Sarvnaz Karimi, and Timothy Baldwin. 2010. Evaluating topic models for digital libraries. In Proceedings of the 10th Annual foint Conference on Digital Libraries. ACM, 215-224.

[50] David Newman, Padhraic Smyth, Max Welling, and Arthur U. Asuncion. 2008. Distributed inference for latent dirichlet allocation. In Proceedings of the Advances in Neural Information Processing Systems. 1081-1088.

[51] Dat Quoc Nguyen, Richard Billingsley, Lan Du, and Mark Johnson. 2015. Improving topic models with latent feature word representations. Transactions of the Association for Computational Linguistics 3 (2015), 299-313.

[52] Srivastava Nitish, Ruslan Salakhutdinov, and Geoffrey E. Hinton. 2013. Modeling documents with a deep boltzmann machine. In Proceedings of the 29th Conference on Uncertainty in Artificial Intelligence (UAI'13). AUAI Press, Arlington, Virginia, 616-624.

[53] Matthew E. Peters, Mark Neumann, Mohit Iyyer, Matt Gardner, Christopher Clark, Kenton Lee, and Luke Zettlemoyer. 2018. Deep contextualized word representations. In Proceedings of the 2018 Conference of the North American Chapter of the Association for Computational Linguistics: Human Language Technologies.

[54] Jim Pitman and Marc Yor. 1997. The two-parameter poisson-dirichlet distribution derived from a stable subordinator. Annals of Probability 25, 2 (1997), 855-900.

[55] Yong Ren, Yining Wang, and Jun Zhu. 2017. Spectral learning for supervised topic models. IEEE Transactions on Pattern Analysis and Machine Intelligence 40, 3 (2017), 726-739. 
[56] Alexander M. Rush, Sumit Chopra, and Jason Weston. 2015. A neural attention model for abstractive sentence summarization. Proceedings of the 2015 Conference on Empirical Methods in Natural Language Processing (2015), 379-389.

[57] Masa-Aki Sato and Shin Ishii. 2000. On-line EM algorithm for the normalized Gaussian network. Neural Computation 12, 2 (2000), 407-432.

[58] Iulian V. Serban, Alessandro Sordoni, Yoshua Bengio, Aaron Courville, and Joelle Pineau. 2016. Building end-to-end dialogue systems using generative hierarchical neural network models. In Proceedings of the 13th AAAI Conference on Artificial Intelligence. 3776-3783.

[59] Yelong Shen, Xiaodong He, Jianfeng Gao, Li Deng, and Grégoire Mesnil. 2014. Learning semantic representations using convolutional neural networks for web search. In Proceedings of the 23rd International Conference on World Wide Web. 373-374.

[60] Alexander Smola and Shravan Narayanamurthy. 2010. An architecture for parallel topic models. Proceedings of the VLDB Endowment 3, 1-2 (2010), 703-710.

[61] Nitish Srivastava and Ruslan Salakhutdinov. 2012. Learning representations for multimodal data with deep belief nets. In Proceedings of the International Conference on Machine Learning Workshop. Vol. 79.

[62] Nitish Srivastava and Russ R. Salakhutdinov. 2012. Multimodal learning with deep boltzmann machines. In Proceedings of the Advances in Neural Information Processing Systems. 2222-2230.

[63] Keith Stevens, Philip Kegelmeyer, David Andrzejewski, and David Buttler. 2012. Exploring topic coherence over many models and many topics. In Proceedings of the 2012 Joint Conference on Empirical Methods in Natural Language Processing and Computational Natural Language Learning. Association for Computational Linguistics, 952-961.

[64] Ilya Sutskever, James Martens, and Geoffrey E. Hinton. 2011. Generating text with recurrent neural networks. In Proceedings of the 28th International Conference on Machine Learning. 1017-1024.

[65] Duyu Tang, Bing Qin, and Ting Liu. 2015. Document modeling with gated recurrent neural network for sentiment classification. In Proceedings of the 2015 Conference on Empirical Methods in Natural Language Processing. 1422-1432.

[66] Jian Tang, Zhaoshi Meng, Xuanlong Nguyen, Qiaozhu Mei, and Ming Zhang. 2014. Understanding the limiting factors of topic modeling via posterior contraction analysis. In Proceedings of the International Conference on Machine Learning. 190-198.

[67] Yee Whye Teh, Michael I. Jordan, Matthew J. Beal, and David M. Blei. 2006. Hierarchical dirichlet processes. fournal of the American Statistical Association 101, 476 (2006), 1566-1581.

[68] Martin J. Wainwright and Michael I. Jordan. 2008. Graphical models, exponential families, and variational inference. Foundations and Trends in Machine Learning 1, 1-2 (2008), 1-305.

[69] Hanna M Wallach, Iain Murray, Ruslan Salakhutdinov, and David Mimno. 2009. Evaluation methods for topic models. In Proceedings of the 26th Annual International Conference on Machine Learning. ACM, 1105-1112.

[70] Xiaolong Wang, Ross Girshick, Abhinav Gupta, and Kaiming He. 2018. Non-local neural networks. In Proceedings of the IEEE Conference on Computer Vision and Pattern Recognition. 7794-7803.

[71] Jônatas Wehrmann, Gabriel S. Simões, Rodrigo C. Barros, and Victor F. Cavalcante. 2018. Adult content detection in videos with convolutional and recurrent neural networks. Neurocomputing 272 (January 2018), 432-438.

[72] Xing Wei and W. Bruce Croft. 2006. LDA-based document models for ad-hoc retrieval. In Proceedings of the 29th Annual International ACM SIGIR Conference on Research and Development in Information Retrieval. 178-185.

[73] Guoqing Xiao, Kenli Li, and Keqin Li. 2017. Reporting 1 most influential objects in uncertain databases based on probabilistic reverse top-k queries. Information Sciences 405 (September 2017), 207-226.

[74] Mingyang Xu, Ruixin Yang, Steve Harenberg, and Nagiza F. Samatova. 2017. A lifelong learning topic model structured using latent embeddings. In Proceedings of the 2017 IEEE 11th International Conference on Semantic Computing (ICSC'17). IEEE, 260-261.

[75] Min Yang, Tianyi Cui, and Wenting Tu. 2015. Ordering-sensitive and semantic-aware topic modeling. In Proceedings of the 29th AAAI Conference on Artificial Intelligence. 2353-2359.

[76] Ke Zhai, Jordan Boyd-Graber, Nima Asadi, and Mohamad L. Alkhouja. 2012. Mr. LDA: A flexible large scale topic modeling package using variational inference in mapreduce. In Proceedings of the 21st International Conference on World Wide Web. ACM, 879-888.

[77] Mingyuan Zhou, Yulai Cong, and Bo Chen. 2016. Augmentable gamma belief networks. The fournal of Machine Learning Research 17, 1 (2016), 5656-5699.

[78] Xu Zhou, Kenli Li, Yantao Zhou, and Keqin Li. 2015. Adaptive processing for distributed skyline queries over uncertain data. IEEE Transactions on Knowledge and Data Engineering 28, 2 (2015), 371-384.

Received December 2018; revised February 2020; accepted July 2020 